\title{
烯丙基肟的环化反应合成异噁唑啉衍生物的研究进展
}

\author{
刘颖杰* 孟建萍李晨林立青 许颖* \\ (哈尔滨商业大学药学院 哈尔滨 150076)
}

\begin{abstract}
摘要 异噁唑啉是一类具有诸多生物学特性的杂环, 是许多天然产物和生物活性化合物的关键结构骨架, 有效的异噁 唑啉合成方法已成为广泛的研究主题. 描述了利用烯丙基肜合成各种官能化异噁唑啉的最新研究进展, 其涉及在氧化 剂存在下的自由基氧化环化反应. 这些反应通常使用容易获得的氧化剂和不同的金属或无金属作为催化剂，在中性反 应条件下进行反应.
\end{abstract}

关键词 异噁唑啉; 烯丙基肜; 环化反应; 金属催化

\section{Progress in the Synthesis of Isoxazoline Derivatives by Cycloylation of Allyl Oxime}

\author{
Liu, Yingjie* Meng, Jianping Li, Chen Lin, Liqing $\quad \mathrm{Xu}$, Ying* \\ (School of Pharmacy, Harbin University of Commerce, Harbin 150076)
}

\begin{abstract}
Isoxazoline is an important class of heterocyclic ring with a wide range of biological characteristics, which is the key structural framework of many natural products and bioactive compounds. The effective synthetic method of isoxazoline has been the subject of extensive research. In this review, the latest progress in the synthesis of various functionalized isoxazoline by allyl oxime, involving the radical oxidation/cyclization reaction in the presence of oxidants is described. These reactions are usually carried out under neutral conditions using readily available oxidants and different metals or metal-free catalysis.
\end{abstract}

Keywords isoxazoline; allyl oxime; cyclization reaction; metal catalysis

肜的结构通式是 $\mathrm{RR}^{\prime}=\mathrm{NOH}$ (其中 RR'代表烃基), 广泛应用于化学领域, 如有机化学和生物化学等 ${ }^{[1]}$. 烯 丙基朊是一类活性很强、作用广泛的中间体化合物(图 1a), 因此可以利用烯丙基肜合成各种异噁唑啉类衍生 物 ${ }^{[2]}$. 杂环化合物在化学工业的许多领域中起着重要作 用, 包括农用化学品和染料的开发 ${ }^{[3-4]}$. 此外, 在 $70 \%$ 的 市售药物和 $80 \%$ 的消耗最多药物中, 至少有一个杂环单 位 ${ }^{[5-6]}$. 异噁唑啉和相应的不饱和类似物异噁唑是一类 重要的杂环化合物(图 1b), 常出现在多种化合物中, 包 括生物活性体、手性配体、有机合成中间体等, 可以广 泛应用于各种生物活性物质和多用途前体中 ${ }^{[7]}$, 且在药 物发现中发挥着越来越重要的作用 ${ }^{[8]}$. 带有异噁唑啉结 构的药物具有抗菌活性、杀菌活性、灭蚊活性、抗阿尔 茨海默病活性、抗癌活性、抗炎症活性、镇静作用以及<smiles></smiles>

(a)<smiles>[R]CC1ON=C([R])C1([R])[R]</smiles>

(b)

\section{图 1 烯丙基肟和异噁唑啉结构}

Figure 1 Basic structure of isoxazoline and allyl oxime 中枢神经系统作用等 ${ }^{[9-14]}$. 例如, 异噁唑啉衍生物(1)是 一种血小板糖蛋白IIb/IIIa受体怙抗剂 ${ }^{[15]}$, 而而 4,5 -二氢3-(4-羟基苯基)-5-异噁唑乙酸甲酯(2)则是炎性因子MIF 的抑制剂 ${ }^{[16]}$, 鬼臼毒素(3)和(4)主要是杀虫和抗菌活性 比较强, 近年来葡萄糖衍生的螺苷一异噁唑啉通过抑制 糖原磷酸化酶 GP (5) 被报道为 2 型糖尿病的降糖药 ${ }^{[17]}$, COX-2抑制剂Parecoxid (6)和Valdecoxib (7)是新型非甾

\footnotetext{
* Corresponding authors. E-mail: liuyj691@nenu.edu.cn; 894132290@qq.com Received March 24, 2020; revised May 3, 2020; published online May 27, 2020. Project supported the Natural Science Foundation of Heilongjiang Province (No. YQ2019B004), the Youth Reserve Talent Program of Harbin University of Commerce (No. YQ2019CX36) and the Youth Innovation Talent Project of Harbin University of Commerce (No. 2016QN056). 黑龙江省自然科学基金(No. YQ2019B004)、哈尔滨商业大学青年后备人才(No. 2019CX36)及哈尔滨商业大学青年创新人才(No. 2016QN056)资助项目.
} 
体类抗炎药(图2), 具有较强的抗炎镇痛作用 ${ }^{[18]}$. 异噁唑 环是市场上最常用的骨架结构之一 ${ }^{[19]}$, 如邻氯西林、双 氯西林、氟氯西林、啞西林等, 侧链上有3-芳基异啞唑 基. 此外, 异啞唑啉和异噁唑可作为合成 $\beta$-氨基酸 ${ }^{[20]} \beta-$ 羟基酮 ${ }^{[21]}$ 和氨基醇 ${ }^{[22]}$ 的重要前体.

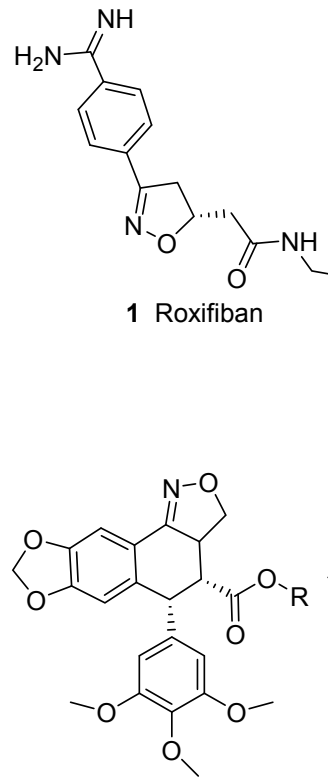

3 Zolipodophyllol

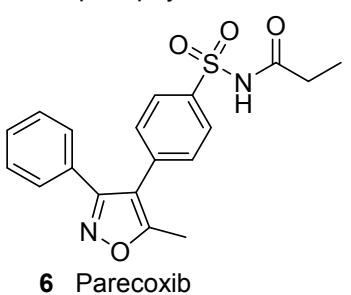

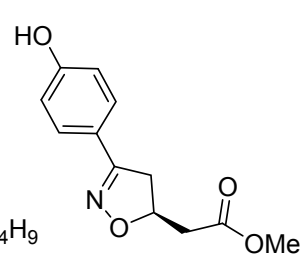

2 ISO-1<smiles>[R]C1=NOC(C(=O)O[C@H]2c3cc4c(cc3[C@@H](c3cc(OC)c(OC)c(OC)c3)[C@H]3C(=O)OC[C@@H]23)OCO4)C1</smiles>

4 Podophyllotoxin

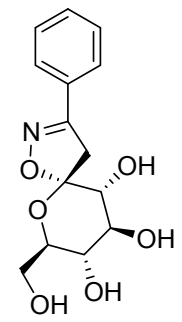

5 GP inhibitor

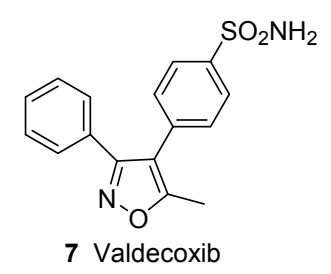

图 2 异噁唑啉衍生物或含有异啞唑啉基团的活性分子

Figure 2 Isoxazoline derivatives or active molecules containing isoxazoline groups

\section{2 异噁唑啉衍生物的合成}

由于异噁唑啉广泛的生物活性, 现在已经有很多方 法来合成异噁唑啉类化合物. 其中, 最常见的合成方法 是烯烃或是炔烃与腈的氧化物的 1,3-环加成反应 ${ }^{[23-24]}$ (Scheme 1), 腈的氧化物一般是由取代的羰基 ${ }^{[25]}$ 或是硝 基化合物 ${ }^{[26]}$ 原位制备的, 这一合成方法也是制备异啞 唑啉衍生物的首选方法.

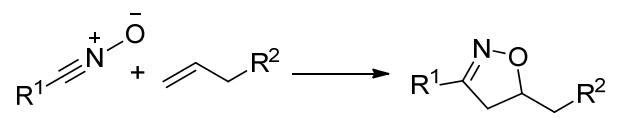

图式 1 腈氧化物与烯烃环加成合成异噁唑啉

Scheme 1 Synthesis of isoxazoline by cycloaddition of nitrile oxides and olefins

2012 年, 韩丙课题组 ${ }^{[27]}$ 基于烯丙基肟为原料, 催化

环化合成了异噁唑啉衍生物, 在过去这一方法并不常 用. 通常情况下, 烯丙基肟的环化反应按反应机理大致 可分为以下三类: (1)烯键先发生亲电加成或被亲电试剂 活化，肟羟基再在分子内亲核进攻; (2)肟羟基首先被氧 化成氮氧自由基，再经自由基环合反应生成异啞唑啉; (3)自由基对双键加成产生碳自由基，后者被氧化成碳 正离子, 再进行碳氧键偶联或是产生的碳自由基与金属 偶联，经还原消除生成产物. 这一环化反应过程中催化 剂、氧化剂、温度以及是否在有氧环境等条件对反应影 响很大，根据反应条件可以将合成方法分为金属催化、 非金属催化和绿色催化三类.

\section{1 基于金属催化合成异噁唑啉}

金属催化是比较常用的一种方法，使用多种金属催 化剂(钯、铜、锰等), 从而实现了烯丙基肜的氧化环化.

\section{1 .1 以金属钯为催化剂合成异噁唑啉}

过渡金属催化的交叉偶联反应是现代有机合成和 药物合成中的有效手段. 近年来, 以钯为催化剂激活碳 碳双键，从肜侧引发反应，参与分子内加成的肜基为合 成异噁唑啉衍生物提供了一种简便的方法 ${ }^{[28]}$.

2008 年, 陈元伟等 ${ }^{[29]}$ 提出了一种新的合成异啞唑 啉衍生物的方法，不饱和肪通过钯催化芳基溴化物得到 3,5-双取代异噁唑，在反应过程中，使用配体 Xantphos 这一步至关重要(Scheme 2).

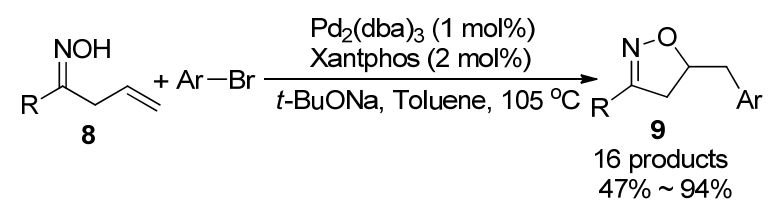

图式 2 钯催化不饱和肟合成异噁唑啉

Scheme 2 Synthesis of isoxazoline by palladium-catalyzed unsaturated oximes

2010 年, Loh 等 ${ }^{[30]}$ 研究报道了一种钯催化的肜, 以 $101 \mathrm{kPa}$ 的空气为唯一氧化剂(Scheme 3), 辅助烯烃的分 子内双加氧反应. 在温和的条件下, 将大气中的 $\mathrm{O}_{2}$ 掺入 到合成过程中.

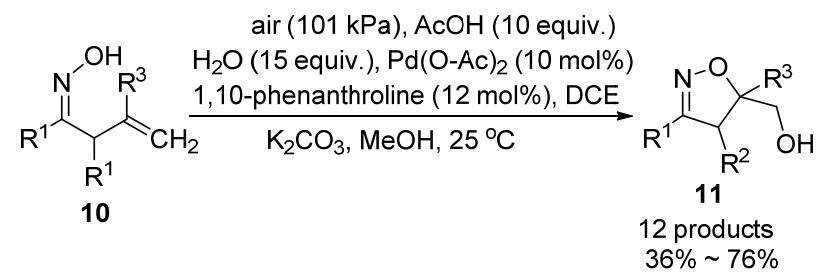

图式 3 钯催化烯烃分子内双加氧反应

Scheme 3 Palladium-catalyzed intramolecular oxidation of olefins

2014 年, 朱晨课题组 ${ }^{[31}$ 报道了肟介导的 5-甲基异 
啞唑的快速获得及其在奥拉西林合成中的应用, 产率中 等至良好(Scheme 4). 这一新方法的实用性在具有生物 活性的戊二肜和奥拉西林的快速合成中得到了很好的 证明.
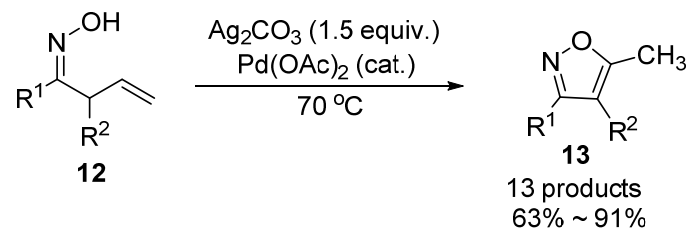

图式 4 钯催化不饱和肜氧化环化

Scheme 4 Palladium-catalyzed oxidation cyclization of unsaturated oximes

\section{1 .2 金属锰为催化剂合成异嚊唑啉}

分子氧是一种理想的氧化剂, 因为它丰富、便宜、 对环境无害 ${ }^{[32]}$. 在分子氧存在的情况下, 过渡金属催化 和无金属氧化双官能化的未激活的 $\mathrm{C}=\mathrm{C}$ 键, 使分子氧 直接与底物结合, 这已经成为一个有吸引力且强大的策 略. 最近, 报道了几种合成 4,5-二氢异噁唑啉醇的新方

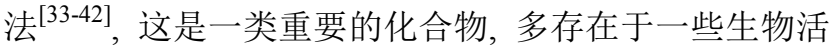
性物质 ${ }^{[43]}$ 和有机合成的中间体中 ${ }^{[44]}$.

2016 年, Yamamoto 等 ${ }^{[45]}$ 研究发现用锰促进氧化环 化的反应来制备 4,5-二氢异噁唑啉醇(Scheme 5). 该反 应中的所有操作都是在没有冷却、加热或高压条件的空 气中进行. 在不加入其他添加剂的情况下, 金属锰可以 通过吸收分子氧来促进氧化环化反应 ${ }^{[44,46]}$, 将分子氧作 为氧化剂为制备 4,5-二氢异噁唑啉提供了一种方法, 该 方法具有操作简单、底物适用范围广、官能团具有很好 的兼容性及温和环保等优点.<smiles>[R]C(=C)/C([R])=N/O</smiles>

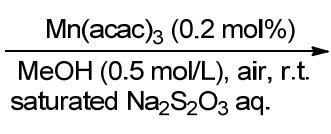

图式 5 锰催化氧化合成 4,5-二氢异噁唑啉 Scheme 5 Synthesis of 4,5-dihydroisoxazoline by manganese catalysis

\subsection{3 铜催化合成异噁唑啉}

芳基烷基砜是一类重要的化合物，广泛应用于医 药、生物活性产品和材料等领域 ${ }^{[47]}$, 同时也是用途广泛 的合成中间体 ${ }^{[48]}$. 传统上, 芳基烷基砜的合成是通过亲 核试剂与亚硫酸钠或硫酚的亲核取代, 然后进行氧化反 应. 2016 年, Gao 等 ${ }^{[49}$ 研究出一种通过铜催化烯醇酸盐 与亚磺酸钠的氧磺酰化反应来生成磺酰化环醚, 通过 $m$-CPBA 的氧化反应生成相应的砜衍生物. 此外, 砜试 剂可以用于砜取代杂环化合物的合成.
2017 年，王力竞及其同事 ${ }^{[50]}$ 提出用烯烃和砜试剂 直接合成砜取代杂环化合物的方法, 以铜为催化剂, 引 入砜基对异啞唑啉进行改性，通过铜介导的未活化烯烃 肜与底物亚磺酸钠的氧磺酰化反应合成磺化异啞唑啉 (Scheme 6). 该反应在室温下很容易进行，而且产率良 好.

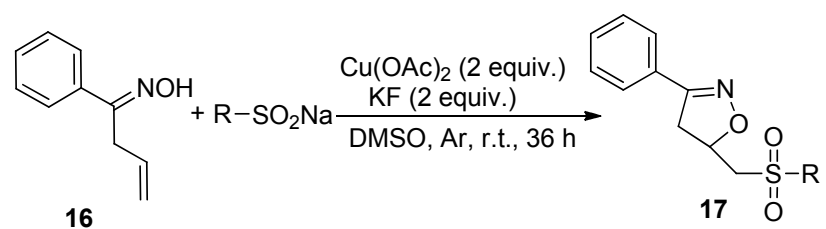

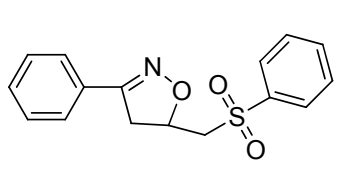

$17 a, 80 \%$
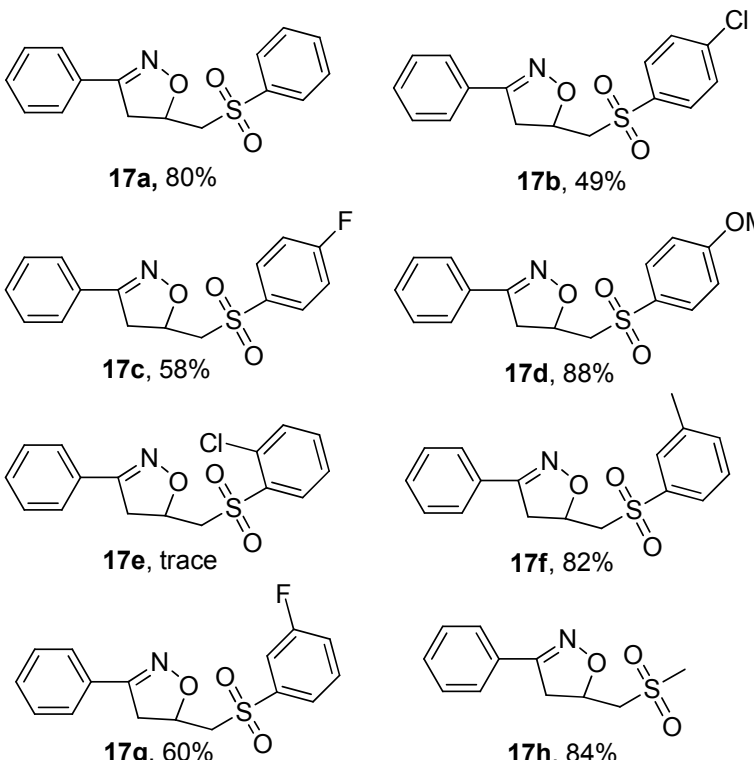

$17 b, 49 \%$
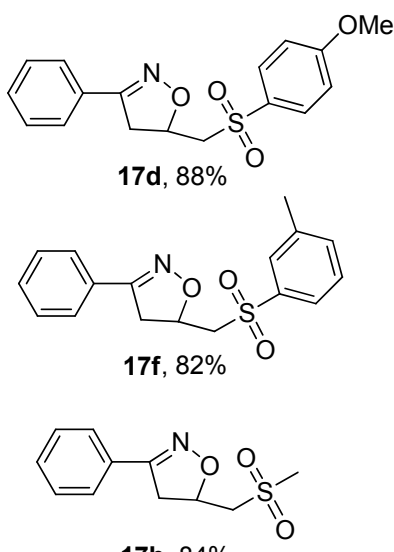

$17 \mathrm{~h}, 84 \%$

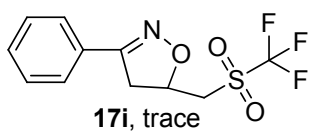

图式 6 铜催化不饱和肟的氧磺酰化反应

Scheme 6 Oxidative sulfonylation of unsaturated oximes catalyzed by copper

氧磺酰化反应机制如 Scheme 7, 最初, 肜 16 和 KF 反应生成中间产物 18. 根据文献[51]可知，中间产物 $\mathbf{1 8}$ 被氧化为相应的肟基自由基 19. 同时, 磺酸钠被铜氧化 生成磺酰自由基 21, 第二次氧化生成磺酰阳离子 22. 在 路径 a 中, 烯烃 18 被亲电磺酰阳离子 22 激活, 形成中 间产物 23, 然后经过环化反应生成所需的异噁唑啉 17. 在路径 b 中, 磺酰自由基 21 与肟基 19 反应生成所需产 物 17 .

上述所解释的机理可能难以理解, 后来查阅相关文 献发现，吕允贺等 ${ }^{[52-55}$ 提出了一种更为容易理解接受 的反应机制(Scheme 8). 首先, 磺酸钠 20 在二价铜作用 下生成磺酰自由基 $\mathbf{2 1}$, 磺酰自由基 $\mathbf{2 1}$ 加成到肟 $\mathbf{1 8}$ 的双 键上生成碳中心自由基的 24, 24 再与二价铜作用生成中 


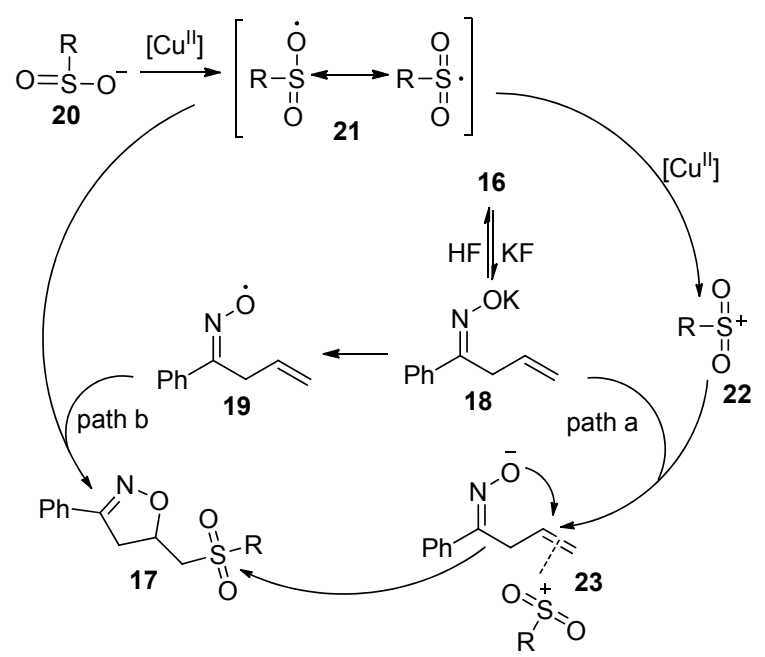

图式 7 氧磺酰化反应机制

Scheme 7 Mechanism of oxygen sulfonation

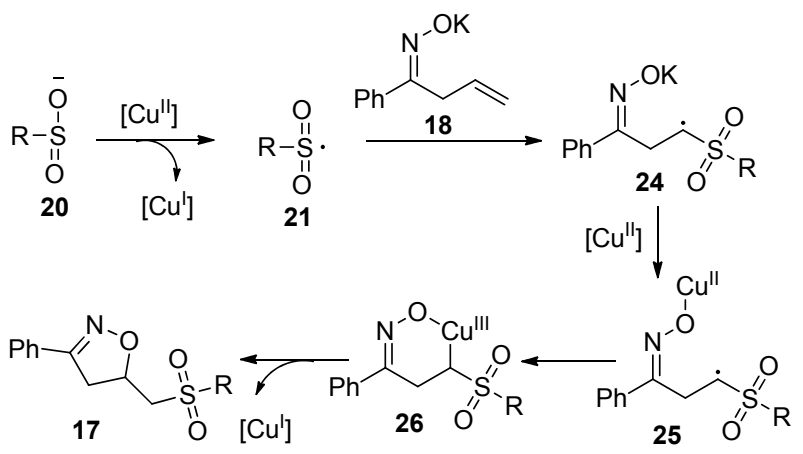

图式 8 氧磺酰化反应机制

Scheme 8 Mechanism of oxygen sulfonation

间体 25 , 然后 25 中的二价铜离子与碳自由基成键生成 三价铜中间体 26, 最后 26 还原消除, 脱去一价铜生成
所需的异噁唑啉衍生物 17.

2018 年，刘心元等 ${ }^{[56}$ 报道了一种铜催化不对称氧 三氟甲基化的烯烃肜和 Togni's 试剂的反应研究(Scheme 9), 该反应为合成含有 $\alpha$-立体中心的异噁唑啉类化合物 提供了直接的途径, 最终生成含有 $\mathrm{CF}_{3}$ 的异噁唑啉的高 度对映选择性结构, 且有着良好的产率和选择性.

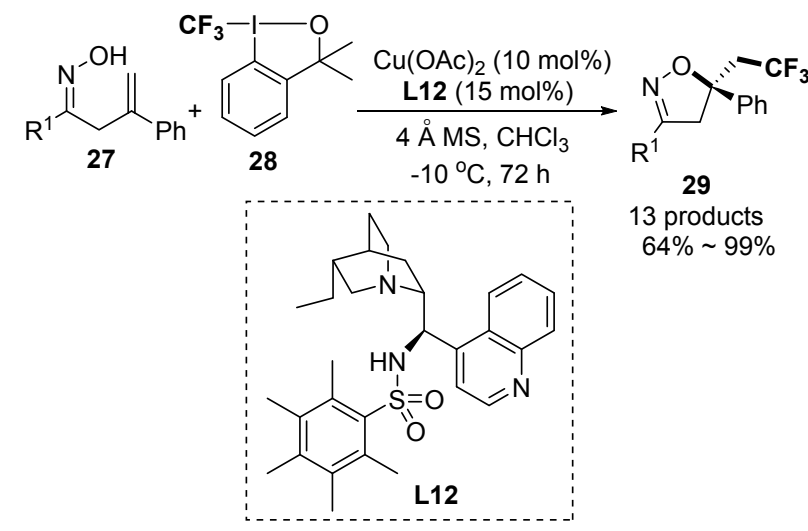

图式 9 铜催化烯烃弜的三氟甲基化反应

Scheme 9 Trifluoromethylation of alken oximes catalyzed by copper

根据反应和过去的研究 ${ }^{[57-58]}$ 提出这一反应的发生 机制(Scheme 10)，原位形成的双螯合型 $\mathrm{Cu}^{\mathrm{I}}$ 络合物 $\mathbf{3 0}$ 通 过配体交换, 可以作为一种活性催化剂, 与 28 进行最初 单电子转移, 生成 $\mathrm{Cu}^{\mathrm{II}}$ 络合物 31 和 $\mathrm{CF}_{3}$ 自由基. 后者迅 速与烯烃进行反应生成自由基中间体 32, 32 也可被络合 物 31 捕获, 通过配体交换形成 33. 随后的分子内氧化 还原反应生成了 34, 然后还原消除得到最终产物 29 ${ }^{[59]}$. 2.1 .4 钴配合物的氧化环化合成异噁唑啉

异噁唑啉类化合物是有机合成中的重要中间体. 考

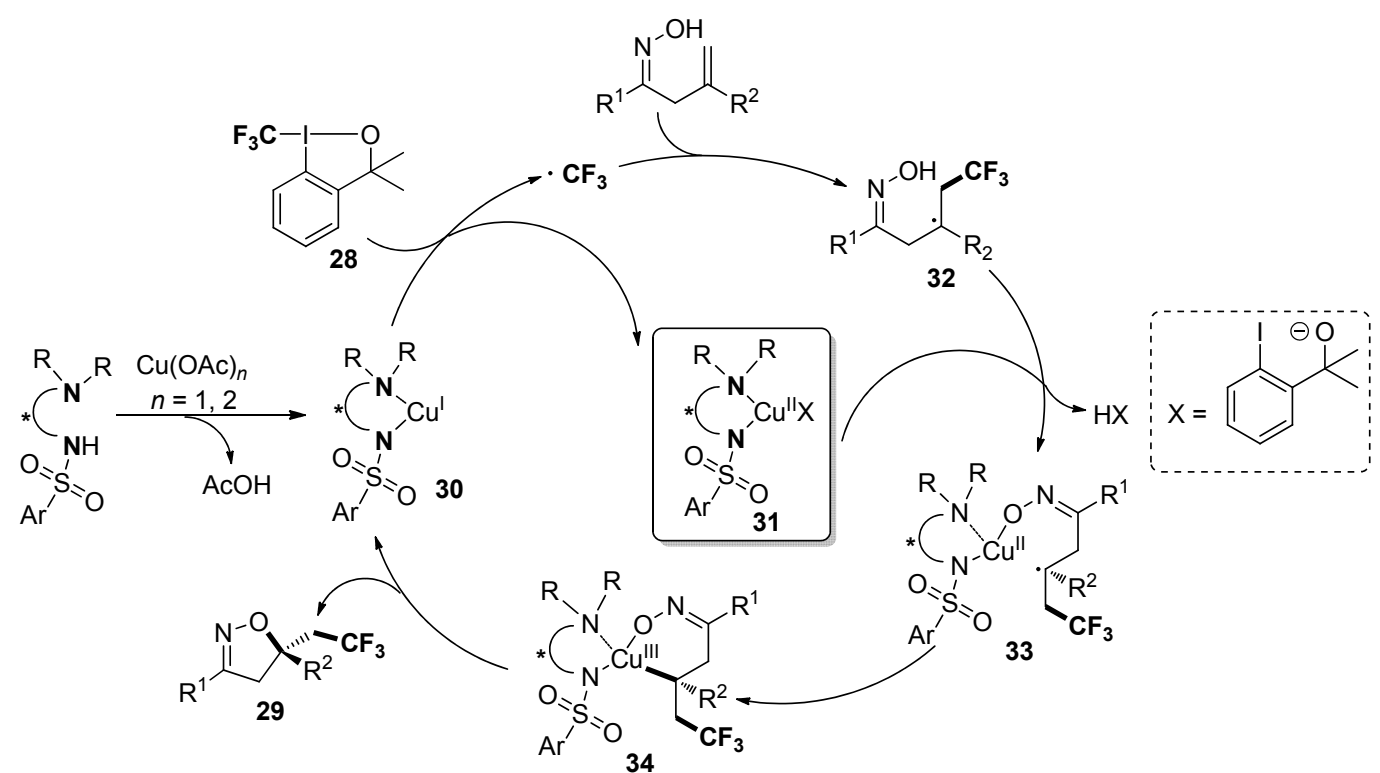

图式 10 铜催化的三氟甲基化反应机制

Scheme 10 Reaction mechanism of copper-catalyzed trifluoromethylation 
虑到钴配合物在烯烃氧化生成中的催化能力, 我们设想 钴催化剂也适用于这种类型的氧化环化. 过渡金属催化 烯烃的好氧氧化不仅是化学工业中的一个重要过程, 在 当前的有机合成中也具有重要的应用价值 ${ }^{[60]}$. Mukaivama 等 ${ }^{\left[{ }^{[1]}\right.}$ 的研究已经证明钴配合物可以有效催化烯烃 在还原剂(通常是作为溶剂的二次醇)作用下的好氧氧 化.

2013 年, 于炜等 ${ }^{[62]}$ 检测了几种钴盐和配合物的作 用, 发现 $\mathrm{Co}(\mathrm{nmp})_{2}$ 对促进反应非常有效(Scheme 11). 以 钴络合物 $\mathrm{Co}(\mathrm{nmp})_{2}$ 为催化剂, 可以选择性地获得氧化 终止产物和还原终止产物. 以 $101 \mathrm{kPa} \mathrm{O}_{2}$ 为氧化剂在 $i-\mathrm{PrOH}$ 中进行反应时, 主要产物为氧化终止产物 36. 另 一方面, 在空气和甲苯中进行反应, 20 equiv.的 1,4-环己 二(CHD)的情况下主要生成还原性终止产物 37. 这种方 法为 4,5-二氢异噁唑的合成提供了一种替代方案.

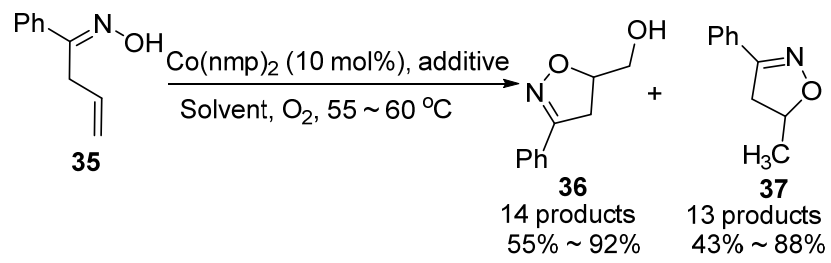

图式 11 钴催化 $\beta, \gamma$-不饱和肟的氧化环化

Scheme 11 Qxidation cyclization of $\beta, \gamma$ - unsaturated oximes catalyzed by cobalt

在不饱和肜催化环化合成异噁唑啉这一过程，所使 用的有毒金属能够产生大量的无机杂质，不仅浪费原料 而且造成环境污染, 所以限制了使用. 为了实现条件温 和、经济、环境友好的目标, 研究人员又研究了非金属 催化的自由基环化反应以及有机催化环化反应.

\section{2 通过非金属催化合成异噁唑啉}

非金属催化的研究报道也很多. 在非金属催化过程 中，通常用 2,2,6,6-四甲基哌啶氧化物(TEMPO)、亚硝酸 叔丁酯(TBN)、偶氮二甲酸二乙(DEAD)或是高价碘配合 物等作为引发剂或氧化剂所介导的环化反应来制备异 噁唑啉类化合物.

\subsection{1 以 $\mathrm{TBN}$ 为引发剂催化合成异噁唑啉}

2014 年, 韩丙等 ${ }^{[63]}$ 提出了一种新的无金属亚胺氧 基参与的亚胺氧基化和亚胺肜化方法(Scheme 12). 该 方法利用亚胺氧基的二官能性, 以亚硝酸叔丁酯(TBN) 作为亚胺氧基的引发剂和碳基捕获剂, 实现了烯烃的泛 双官能化. 利用该方案, 可分别从 $\beta, \gamma$-和 $\gamma, \delta$-不饱和酮肜 中合成 4,5-二氢异噁唑和环状亚硝基.

在室温氩气气氛下, 在 $\mathrm{MeCN}$ 中 $\beta, \gamma$-不饱和酮弜 $\mathbf{3 8}$ 与 $\mathrm{TBN}$ 反应 (Scheme 13), 在 $0.5 \mathrm{~h}$ 内形成无色沉淀, 产 率 96\%，研究证实产物为 41. 虽然反应过程中产生了中

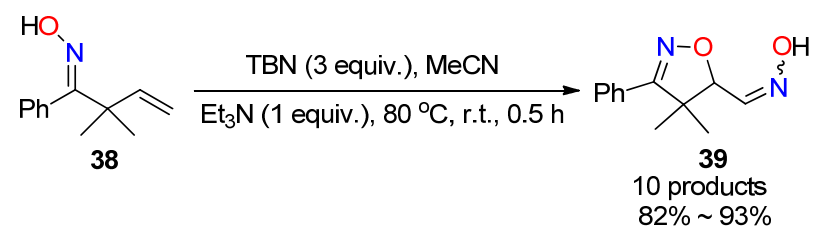

图式 12 亚胺氧基促进烯烃的双官能

Scheme 12 Iminoxy promotes the difunctionalization of alkenes

间体 4,5-二氢异啞唑 40, 但它立即二聚成产物 41. 化合 物 41 在 $80{ }^{\circ} \mathrm{C}$ 的 $\mathrm{MeCN}$ 中受热，最后生成异啞唑啉类化 合物 39

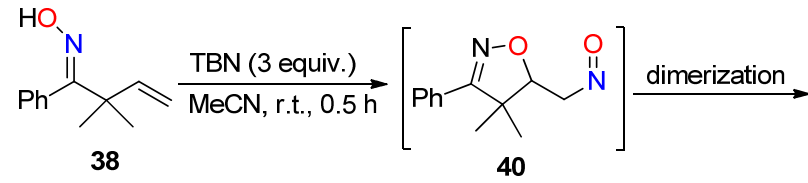

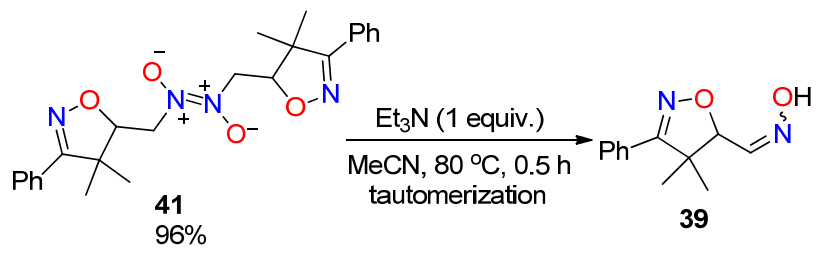

图式 13 亚胺氧自由基促进烯烃的氧化

Scheme 13 Oxidation of alkenes promoted by imide-oxy radicals

\subsection{2 以 TEMPO 和 DEAD 为引发剂催化合成异噁唑啉}

肟基的反应具有很大的合成价值，2012 年，韩丙 等 ${ }^{[27]}$ 提出以酮肟为原料, 使用 TEMPO 或 DEAD 作为引 发剂, 利用此方法分别从 $\beta, \gamma$-和 $\gamma, \delta$-不饱和酮肟通过 $\mathrm{C}-$ $\mathrm{O}$ 和 $\mathrm{C}-\mathrm{N}$ 形成 5-exo-trig 环化反应来合成 4,5-二氢异惡 唑和环硝基.

$\gamma, \delta$-不饱和酮肜由肜基促进 5-exo-trig 氮与碳成键环 化生成环状硝基(Scheme 14) ${ }^{[64]}$
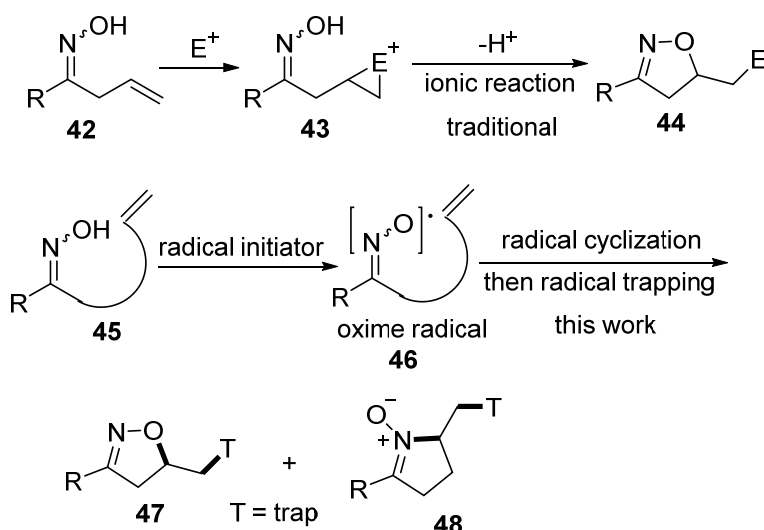

图式 14 肜基的 5-exo-trig 环化

Scheme 14 Cyclozation of 5-exo-trig of the oxime groups 
首先选择 TEMPO 来启动 $\beta, \gamma$-不饱和肜基的自由基 环化. 研究表明, 肜中的氧氢键具有相对较低的解离键 能 ${ }^{[6]}$. 预期这个速度可能通过氢原子抽提(HAT)过程将 肜转化为肜基, 然后形成的肜基经过 5-exo-trig 环化得 到相应的以碳为中心的官能团, 这些官能团可以被迅速 捕获, 从而产生异噁唑啉衍生物 $\mathbf{5 2}$, 其产率为 $71 \%$ (Scheme 15).

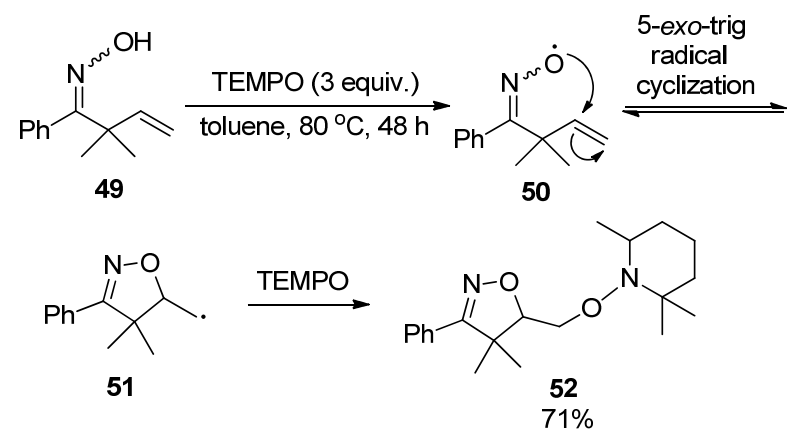

图式 15 TEMPO 催化的 5-exo-trig 环化反应 Scheme 15 5-exo-trig cyclization catalyzed by TEMPO

上述反应所得的产率并不高, 当以 DEAD (1 equiv) 作为肜基引发剂, TEMPO (2 equiv)作为碳基捕获剂时, 得到了相同的产物 $\mathbf{5 2}$, 以及少量的副产物异噁唑啉 $\mathbf{5 3}$ (Scheme 16). 除了异噁唑啉结构基序的重要性外, 化合 物 52 和 53 的形成还分别涉及未活化烯烃的双加氧和氧 化胺化 ${ }^{[66]}$.

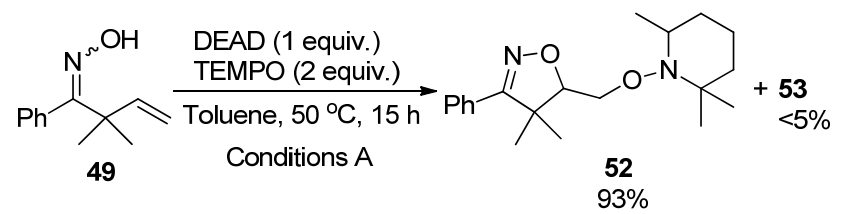<smiles>C=CC(C)(C=C)C(=NO)c1ccccc1</smiles><smiles>CCOC(=O)NN(CC1ON=C(c2ccccc2)C1(C)C)C(=O)OCC</smiles>

$91 \%$

图式 16 DEAD 引发的 5-exo-trig 环化反应

Scheme 16 DEAD-induced 5-exo-trig cyclization

\subsection{3以高价碘配合物介导的氧氟化反应}

异噁唑啉类化合物的骨架存在于多种生物活性物 质中, 并在有机合成中起着多种作用 ${ }^{[67]}$. 高价碘配合物 是一种多用途的氧化试剂, 用于介导有机反应, 包括生 成新的 $\mathrm{C}-\mathrm{C}$ 和 $\mathrm{C}$ 一杂原子键 ${ }^{[68]}$. 由于高价碘化物的低 毒、高稳定性及易于操作等特性, 其已成为有机合成中 的合成氧化剂, 可用于调节烯烃的双官能化, 而不需要 使用金属催化剂 ${ }^{[69]}$. 在药物发现过程中, 氟的引入已成 为候选修饰药物的重要策略 ${ }^{[70]}$. 我们设想通过分子内 的氧氟化反应, 烯烃肜可以环化形成一氟甲基取代的异
噁唑啉.

2015 年，王锐等 ${ }^{[71]}$ 开发了一种高度区域选择性的 无金属化方法来对未激活的末端烯烃进行分子内氧化 氧氟化反应(Scheme 17). 这为一氟甲基取代异噁唑啉 类化合物的制备提供了一种有效的方法.

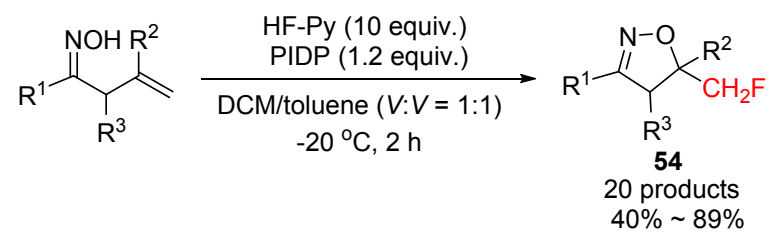

图式 17 碘介导的烯基氧基氟化反应合成异啞唑啉 Scheme 17 Isoxazoline synthesized by iodine-mediated alkenyl oxy fluorination

氧化氟化反应的机制途径见 Scheme 18. 开始, PIDP 与 HF-Py 原位反应, 通过配体交换形成 $\mathrm{PhIF}_{2}$. 然 后烯烃与 $\mathrm{PhIF}_{2}$ 反应生成碘离子 $\mathbf{5 8}$, 碘离子 $\mathbf{5 8}$ 被 $\mathrm{F}$ 离子 攻击形成中间产物 59. 随后发生分子内亲核攻击 $\mathrm{OH}$, 还原消除得到产物 60 .

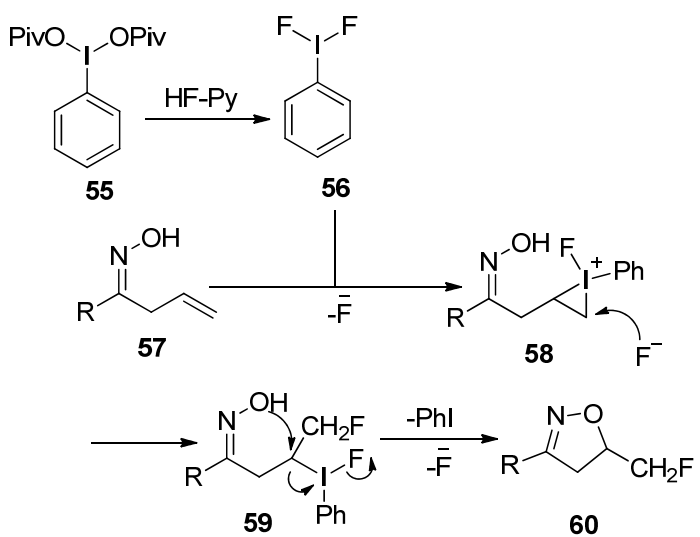

图式 18 氟化反应机制

Scheme 18 Fluorination mechanism

2018 年,蔡春课题组 ${ }^{[72]}$ 提出了一种新型、高效的以 双硫醚和双硒醚为硫和硒源的碘 $(\mathrm{ml})$ 介导的不饱和腙和 肜的分子内酰亚胺和硒官能化反应(Scheme 19). 该反 应为制备异噁唑啉衍生物提供了一种简便、直接的方法. 此外，该方法具有底物适用范围广、官能团兼容性很好、 选择性强等优点.

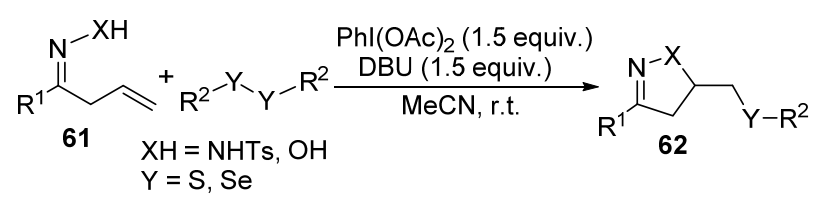

图式 19 碘催化合成异噁唑啉

Scheme 19 Synthesis of isoxazoline catalyzed by iodine 在实验观察的基础上提出了一种机理 ${ }^{[73-76]}, \beta, \gamma$-不 
饱和对苯腙或肟 63 在碱性条件下形成了阴离子中间体 64. 随后, $\mathrm{PhI}\left((\mathrm{OAc})_{2}\right.$ 对 64 进行单电子氧化得到以 $\mathrm{N}$ 或 $\mathrm{O}$ 为中心的自由基 $\mathbf{6 5}$. 自由基 65 在分子内进行自由基 环化反应生成以 $\mathrm{C}$ 为中心的自由基 66 . 自由基 66 与二 苯二硫 $\mathbf{a}$ 反应生成产物 67 , 通过自由基的增殖再生出可 以重新结合成二苯二硫的磺酰基自由基(路径 I). 自由 基 66 也可以与二苯基二烯酰胺 $\mathrm{b}$ 反应生成产物 68 (路径 II) (Scheme 20).

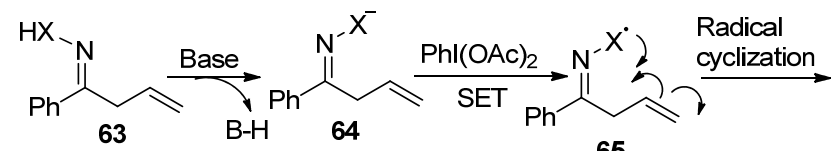
$\mathrm{XH}=\mathrm{NHTs}, \mathrm{OH}$

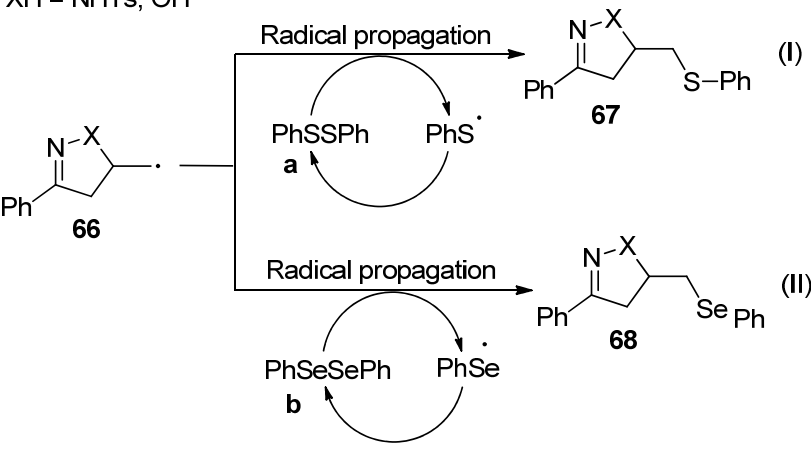

图式 20 碘催化合成异噁唑啉的机理

Scheme 20 Mechanism of synthesis of isoxazoline catalyzed by iodine

2.2.4 由 $t$-BuONO 介导的无金属氧化硝化反应合成异 噁唑啉

2016 年，康彦彪等 ${ }^{[77]}$ 提出了一种由亚硝酸叔丁酯 ( $t$-BuONO)介导的无金属氧化硝化反应(Scheme 21). 在 有氧和无金属条件下，由 $t$-BuONO 介导的烯烃基肜的 自动环化反应. $t$-BuONO 是一种简单的有机 $\mathrm{NO}$ 源, 也 是一种自由基引发剂. 利用分子氧作为唯一的氧化试 剂, 避免了使用如 TEMPO 之类的有机捕获试剂, 硝基 异噁唑啉类化合物产率较高.

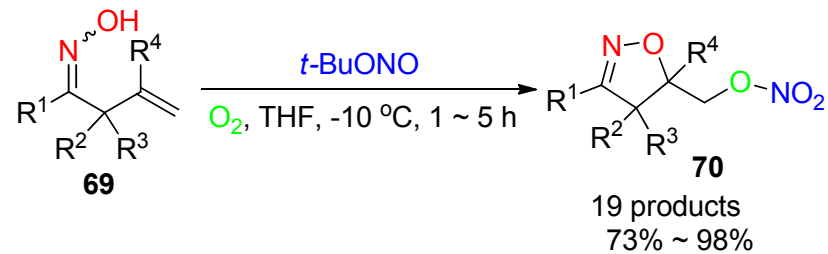

图式 21 由 $t$-BuONO 介导催化合成异噁唑啉 Scheme 21 Synthesis of isoxazoline catalyzed by $t$-BuONO

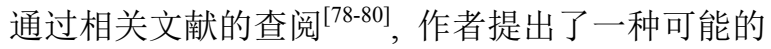
机制来解释烯烃基肜基的自氧化反应(Scheme 22). 肜 71 和 $t$-BuONO 反应产生自由基中间体 72, 然后进行环 化反应生成初级自由基 $\mathbf{7 3}^{[78]}$. 在低温下, 高活性自由基 73 选择性地捕获 $\mathrm{O}_{2}{ }^{[79]}$ 形成过氧自由基 $74^{[80 \mathrm{~b}]}$, 通过捕捉
$\mathrm{NO}$ 来产生过氧亚硝基盐 $\mathbf{7 5}, 75$ 的 $\mathrm{O}-\mathrm{O}$ 裂解重排的发 生提供了环化产物 77

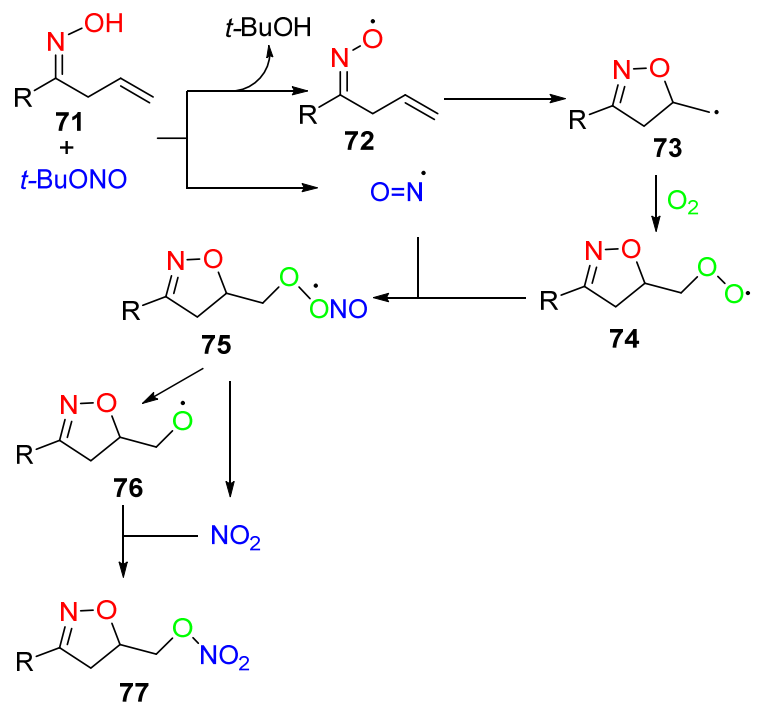

图式 $22 t$-BuONO 介导的氧化硝化反应机制 Scheme 22 Mechanism of t-BuONO mediated nitrification reaction

\section{2 .5 硒和碲原子催化合成异嚊唑啉类化合物}

2019 年, Lenardão 等 ${ }^{[81]}$ 提出了一种制备硫基功能化 异噁唑啉类化合物的新方法(Scheme 23). 该方法包括 $\beta, \gamma$-不饱和肟与亲电硒和碲的反应，在室温条件下 $1 \mathrm{~h}$ 后 可以产生 19 个新的硒和碲的异噁唑啉类化合物, 且收 益良好. 该方法可有效地推广到 5 种新型(双)异啞唑啉 双胞苷的合成, 3-苯基-5-((苯基硒基)甲基)-异啞唑啉是 制备的化合物之一，比塞来昔布具有更好的抗炎和抗水 肿作用。

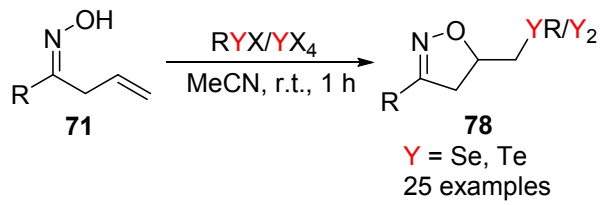

图式 23 含硫异噁唑啉类化合物的合成

Scheme 23 Synthesis of thioisoxazoline compounds

\subsection{6 以 2,2,2-三氟苯乙酮催化氧化合成异噁唑啉}

在 2017 年, Kokotos 等 ${ }^{[82}$ 提出了一种绿色、可持续 的异噁唑啉类化合物的合成方法(Scheme 24). 以 $\mathrm{H}_{2} \mathrm{O}_{2}$ 为氧化剂, 利用 2,2,2-三氟苯乙酮催化氧化烯丙基肜, 为取代异啞唑啉类化合物的合成提供了一种廉价、环保 的方法. 这种合成方法利用廉价的不含金属的有机分子 (2,2,2-三氟苯乙酩)为催化剂和 $\mathrm{H}_{2} \mathrm{O}_{2}$ 为氧化剂, 完成了 烯丙基肟的一锅法环氧化反应，随后进行了原位开环/ 环化反应，以很高产率获得了异噁唑啉类化合物。 

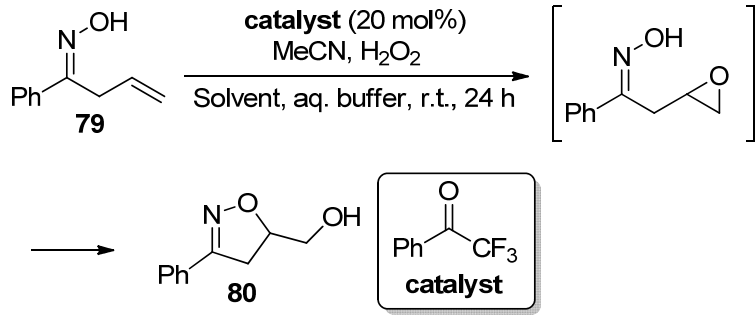

图式 24 烯丙基肪的一锅法环氧化反应

Scheme 24 One-pot epoxidation of allyl oximes

为了解释这一系列的反应过程, 提出了反应机制 (Scheme 25) ${ }^{[83]}$. 最初, 催化剂在水的条件下生成二醇 81, 然后, 在适当的 $\mathrm{pH}$ 下, 乙腈 $\mathrm{MeCN}$ 与 $\mathrm{H}_{2} \mathrm{O}_{2}$ 反应生 成 82,82 与 $\mathrm{H}_{2} \mathrm{O}_{2}$ 一起氧化生成 84 . 另一分子 82 与 84 反应生成了活性氧化剂一环氧化合物烯丙氧肟 79. 最后 脱质子的环氧肟环通过环化作用打开环氧化物, 生成异 噁唑啉 80.

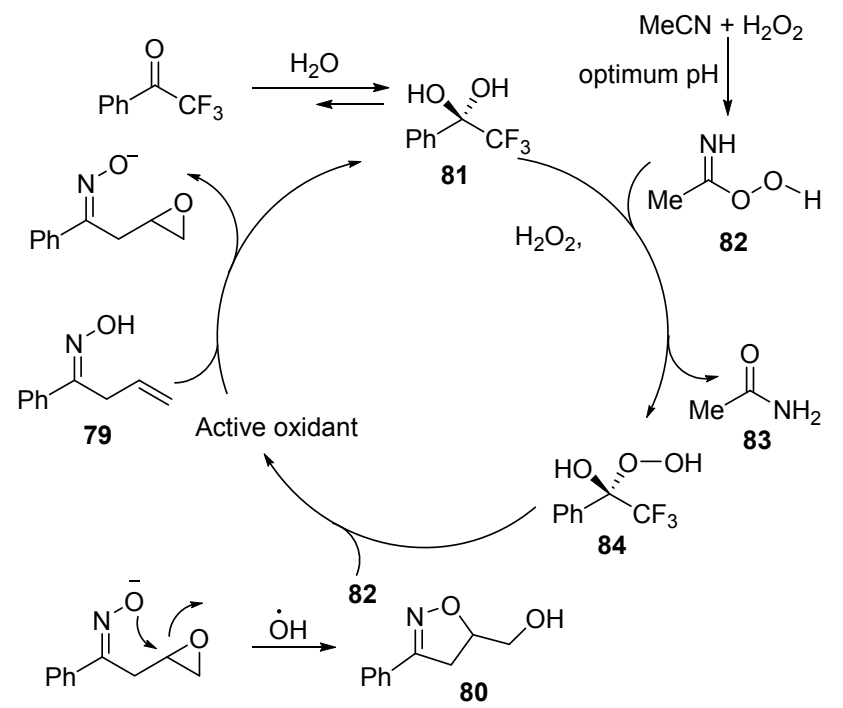

图式 25 一锅法环氧化反应机制

Scheme 25 One pot epoxidation mechanism

2.2.7以三氟异氧尿酸促进烯丙基肜的三氟甲基化 反应合成异噁唑啉

2017 年, 胡雨来课题组 ${ }^{[84}$ 提出以三氟异氰尿酸 (TCCA) 为原料, 利用 $\mathrm{TMSCF}_{3}$ 促进三氟甲基化反应，该 反应具有廉价、实用的优点. TCCA 是一种用途广泛、 无毒的氧化剂或氯化试剂, 工业上稳定性高 ${ }^{[85]}$. 在有机 化学合成领域, TCCA 以其低廉的价格、环境友好的特 性以及参与各种反应的能力, 在很多反应中得到了广泛 的应用 ${ }^{[86]}$. 以 $\mathrm{TMSCF}_{3}$ 为三氟甲基源, TCCA 促进烯丙 基肟类化合物的环化/三氟甲基化反应，为三氟甲基取 代的 4,5-二氢异噁唑的制备提供了一种简洁有效的方法 (Scheme 26).

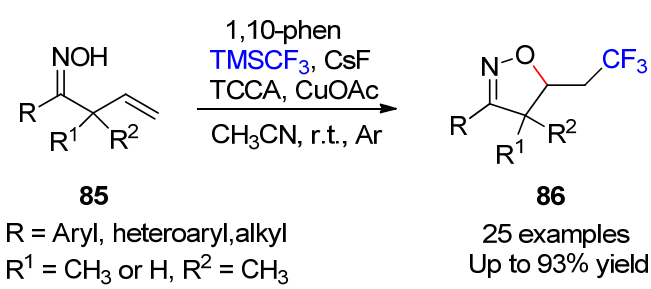

图式 26 三氟异氰尿酸促进烯丙基肜的环化 Scheme 26 Trifluoroisocyanuric acid promoted the cyclization of allyl oximes

这一反应发生的机理如 Scheme 27 所示, 当用 $\mathrm{CuOAc} 、 \mathrm{CsF}$ 和 1,10-菲罗啉与 $\mathrm{TMSCF}_{3}$ 反应时，首先形 成络合物 $87^{[87]}$. 在反应混合物中加入 TCCA 和肜 90 生 成自由基 89 , 从肪 90 的羟基中提取一个质子生成自由 基 91, 最终将 TCCA 转化为异氧尿酸, 并从反应混合物 中进行检测. 自由基 91 的环化得到另一个碳自由基 $\mathbf{9 2}$, 从络合物 87 中提取出一个三氟甲基, 得到最终产物 93.

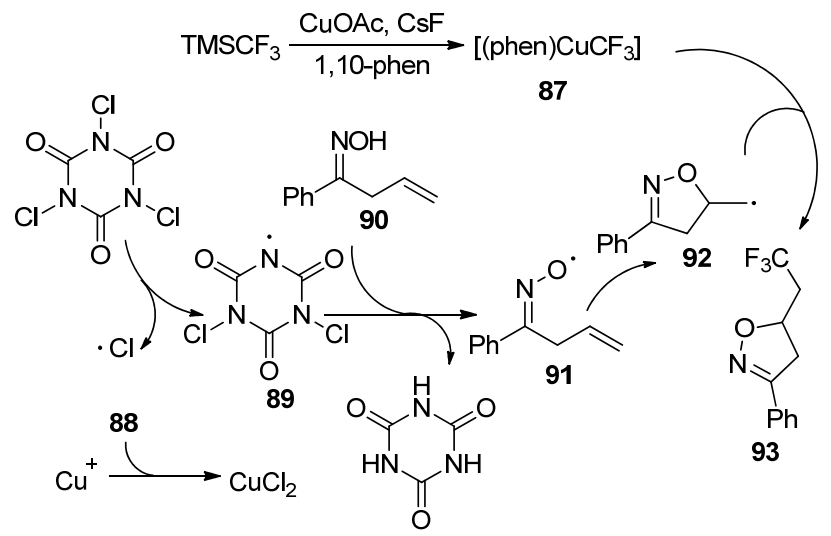

图式 27 三氟甲基化的机制

Scheme 27 Mechanism of trifluoromethylation

非金属催化与金属催化相比，减少了金属试剂使 用，从而避免了有毒金属的危害以及对环境的污染，但 是在非金属催化过程中, 使用了过量的有害氧化剂进行 催化反应，也会对环境造成一定的危害. 因此，为了减 少对环境的污染, 可以采取绿色催化的方法来合成异噁 唑啉衍生物.

\section{3 绿色催化}

近年来, 利用可见光催化不饱和肜从而得到异啞唑 啉衍生物的方法已经开始普及. 使用可见光进行催化, 分子氧作为绿色氧化剂, 避免了使用过量的金属或非金 属氧化剂。

2015 年, 陈加荣课题组 ${ }^{[88]}$ 通过可见光开发了一种 高效温和的光催化自由基三氟甲基化/环化级联反应 (Scheme 28), 该反应能够合成范围广泛的异啞唑啉衍 生物, 产率通常都很高. 


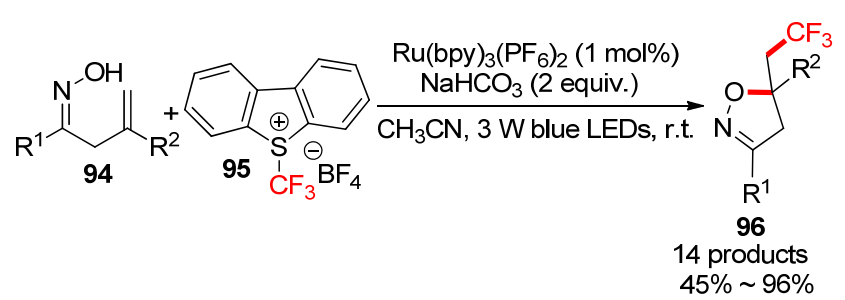

图式 28 光催化 $\beta, \gamma$-不饱和肜

Scheme 28 Photocatalytic $\beta, \gamma$-unsaturated oximes

自由基反应的机理如 Scheme 29 所示. 在可见光照 射下，基态光催化剂 $\left[\mathrm{Ru}(\mathrm{bpy})_{3}\right]^{2+}$ 首先被刺激为激发态 $*\left[\mathrm{Ru}(\mathrm{bpy})_{3}\right]^{2+}$, 然后作为还原剂, 通过固定的过程来还 原试剂 95, 生成高活性的 $\mathrm{CF}_{3}$ 自由基. 随后, $\mathrm{CF}_{3}$ 自由基 在不饱和水合物和肟基的烯烃上进行自由基加成, 得到 新的自由基中间体 99,99 被 $\left[\mathrm{Ru}(\mathrm{bpy})_{3}\right]^{3+}$ 氧化为 $\beta-\mathrm{CF}_{3}$ 自 由基取代的碳正离子中间体 $\mathbf{1 0 0}$ (path a). 但是, 目前还 不能排除另一种通过自由基链增殖, 将 99 自由基转化 为碳正离子 100 的可能性(path b), 最后, 通过亲核环化 形成最终产物 96 或 98.

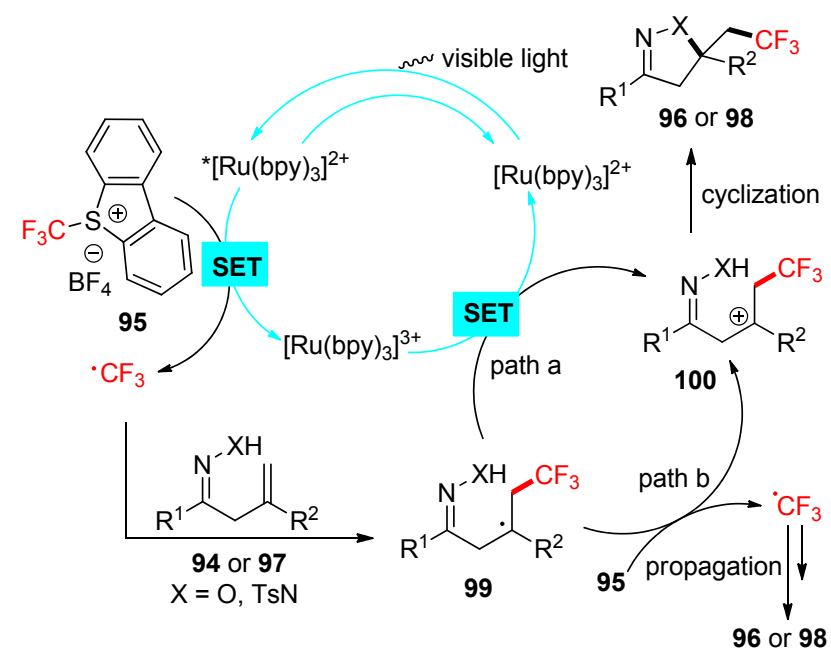

图式 29 自由基反应机制

Scheme 29 Free radical reaction mechanism

近年来，新二氟甲基化试剂和二氟甲基化反应快速 应用和发展 ${ }^{[89]}$ ，自从 Baran 等 ${ }^{[90]}$ 对异芳烃与二氟甲烷磺 酸锌的直接二氟甲基化的开创性工作以来, 已经发展出 几种基于二氟甲基自由基前体，将二氟甲基掺入不同有 机结构的重要方法. 例如, Dolbier 等 ${ }^{[91]}$ 报道了二氟甲基 自由基通过可见光促进的 $\mathrm{CF}_{2} \mathrm{HSO}_{2} \mathrm{Cl}$ 的单电子还原和 构建各种二氟甲基取代的 $N$-杂环的级联反应来获得. Koike 等 ${ }^{[92}$ 报道了 Perylene 催化的一种无金属二氟甲基 化烯烃与磺酸盐反应. 最近, 徐海超课题组 ${ }^{[93]}$ 通过二茂 铁介导的电化学氧化, 从 $\mathrm{CF}_{2} \mathrm{HSO}_{2} \mathrm{NHNHBoc}$ 生成二氟 甲基自由基.
2019 年, 付维军课题组 ${ }^{[94]}$ 提出以含氟砜作为二氟 甲基化和三氟甲基化试剂，成功地实现了 $\beta, \gamma$-不饱和肜 类化合物的可见光催化二氟甲基化/三氟甲基化/环合反 应. 在氧化还原过程中生成相应的二氟甲基异噁唑啉和 三氟甲基异噁唑啉衍生物(Scheme 30). 该方法为二氟 甲基化和三氟甲基化异噁唑啉类化合物的制备提供了 一种选择性的、有吸引力的策略.

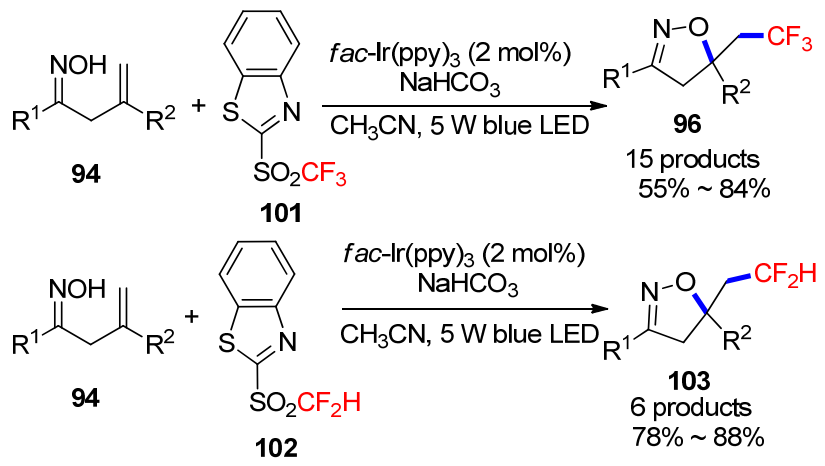

图式 30 可见光诱导的三氟甲基化和二氟甲基化反应 Scheme 30 Visible light induced trifluoromethylation and difluoromethylation

为了解释这一反应，提出了相应的自由基机制 (Scheme 31), 在可见光照射下, 基态的 $\operatorname{Ir}(\mathrm{III})$ 光催化剂 $[\text { faci-ir(ppy })_{3}$ ]处于兴奋状态 $\left[\right.$ faci-ir $\left.(\mathrm{ppy})_{3}{ }^{*}\right]$, 而 $[$ faci$\left.\operatorname{ir}(\mathrm{ppy})_{3}{ }^{*}\right]$ 向氟化砜的单电子转移(SET)产生了 $\operatorname{Ir}(\mathrm{IV})$ 络 合物和氟甲基自由基 104. 104 对 $\beta, \gamma$-不饱和肜 105 进行 自由基加成，生成新的自由基中间体 106, 106 容易被新 形成的 $\operatorname{Ir}(\mathrm{IV})$ 氧化生成碳正离子中间体 107, 同时再生 基态光催化剂 $\left[f a c i-i r(p p y)_{3}\right]$. 最后，在碱基和环合作用 的辅助下，依次进行脱质子反应，形成所需要的产物 108.

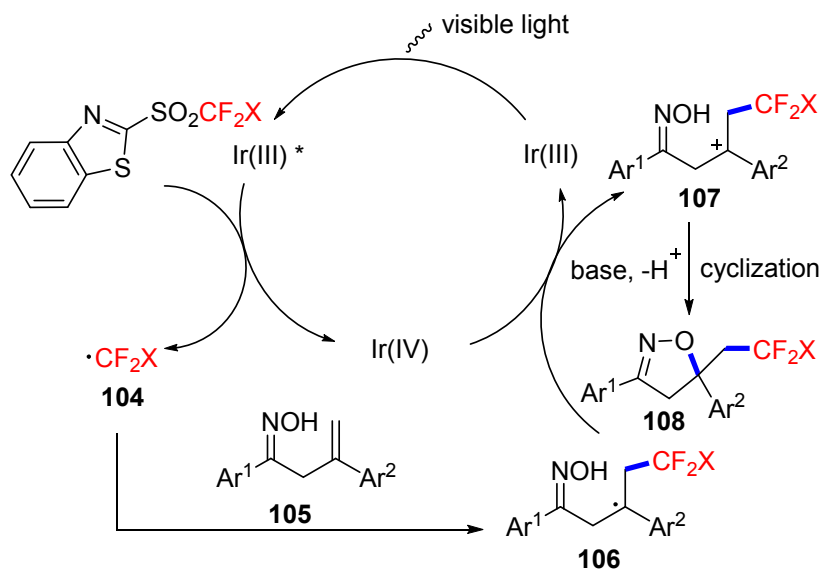

图式 31 自由基反应机制

Scheme 31 Free radical reaction mechanism

2016 年, 陈加荣课题组 ${ }^{[95]}$ 提出采用协同光催化策 略(Scheme 32), 将 $\mathrm{N}-\mathrm{H}$ 和 $\mathrm{O}-\mathrm{H}$ 键直接转化为以 $\mathrm{N}$ 和 
$\mathrm{O}$ 为中心的自由基, 实现了对 $\beta, \gamma$-不饱和肟氧化自由基 氧化胺化和双氧化反应. 在反应中, $\mathrm{O}_{2}$ 不仅作为末端氧 化剂, 而且还作为氧源, 在无金属且温和的反应条件下 合成异噁唑啉衍生物.

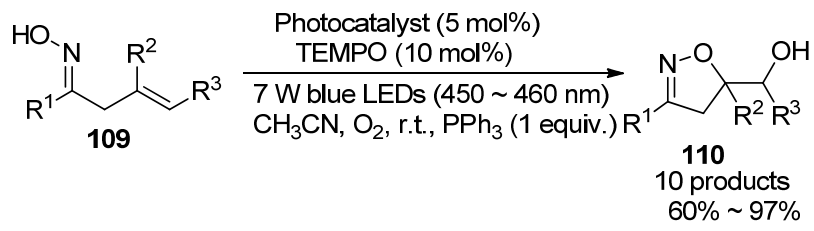

图式 32 协同光催化反应合成异噁唑啉

Scheme 32 Synthesis of isoxazoline by cooperative photocatalytic reaction

\section{3 结论与展望}

近几年来, 基于烯丙基肜自由基催化环化合成异噁 唑啉衍生物的方法取得了极大的进展, 异啞唑啉衍生物 在化工领域、医药行业有着广泛的用途. 但是, 在环化 反应过程中, 涉及到催化剂和氧化剂的使用. 虽然烯丙 基肪进行环化反应合成异噁唑啉衍生物方面取得了很 大进展，但在金属催化过程中会使用大量的金属催化 剂, 对环境的污染比较严重, 而且成本也很高. 非金属 催化中又会用到过量的有害氧化剂, 这些残存的氧化剂 也会对环境产生极大的危害. 因此, 发现更多的绿色环 保、原子利用率高、产率较高的催化方法, 以便实现更 多异噁唑啉衍生物的获得方法, 这样既可以完成催化过 程，又不会对环境造成严重的污染.

\section{References}

[1] (a) Narasaka, K.; Kitamura, M. Eur. J. Org. Chem. 2005, 21, 4505. (b) Kassa, J.; Kuca, K.; Bartosova, L.; Kunesova, G. Curr. Org. Chem. 2007, 11, 267.

[2] For recent selected examples, see: (a) Sato, Y.; Kawaguchi, S.; Nomoto, A.; Ogawa, A. Angew. Chem., Int. Ed. 2016, 55, 9700.

(b) Zhao, J.; Li, P.; Li, X.; Xia, C.; Li, F. Chem. Commun. 2016, 52, 3661 .

(c) Cui, H.; Liu, X.; Wei, W.; Yang, D.; He, C.; Zhang, T.; Wang, H. J. Org. Chem. 2016, 81, 2252.

(d) Lan, X.-W.; Wang, N.-X.; Bai, C.-B.; Lan, C.-L.; Zhang, T.; Chen, S.-L.; Xing, Y. Org. Lett. 2016, 18, 5986.

[3] Pozharskii, A. F.; Katritzky, A. R.; Soldatenkov, A. Heterocycles in Life and Society: An Introduction to Heterocyclic Chemistry, Biochemistry and Applications, John Wiley Sons, New Jersey, 2011, pp. $35 \sim 62$.

[4] Xu, Z.; Ye, T. Heterocycles in Natural Product Synthesis, WileyVCH, Darmstadt, 2011, pp. 459 505.

[5] Alvarez-Builla, J.; Vaquero, J. J.; Barluenga, J. Modern Heterocyclic Chemistry, Wiley-VCH, Darmstadt, 2011, pp. 989 1045 .

[6] Li, J. J.; Hruby, V. Angew. Chem., Int. Ed. 2017, 129, 2583.

[7] Selected examples of biological evaluation of isoxazolines: (a) Castellano, S.; Kuck, D.; Viviano, M.; Yoo, J.; López-Vallejo, F.; Conti, P.; Tamborini, L.; Pinto, A.; Medina-Franco, J. L.; Sbardella, G. J. J. Med. Chem. 2011, 54, 7663.

(b) Poutiainen, P. K.; Venäläinen, T. A.; Peräkylä, M.; Matilainen, J. M.; Väisänen, S.; Honkakoski, P.; Laatikainen, R.; Pulkkinen, J. T.
Bioorg. Med. Chem. 2010, 18, 3437.

(c) Namboothiri, I. N. N.; Rastogi, N. Isoxazolines from Nitro Compounds: Synthesis and Applications, Springer, Berlin, 2008, pp. $1 \sim 44$.

(d) Bode, J. W.; Carreira, E. M. Org. Lett. 2001, 3, 1587.

[8] For selected reviews, see: (a) Rück-Braun, K.; Freysoldt, T. H. E.; Wierschem, F. Chem. Soc. Rev. 2005, 34, 507.

(b) Kaur, K.; Kumar, V.; Sharma, A. K.; Gupta, G. K. Eur. J. Med. Chem. 2014, 77, 121.

(c) Hu, F.; Szostak, M. Adv. Synth. Catal. 2015, 357, 2583.

(d) Morita, T.; Yugandar, S.; Fuse, S.; Nakamura, H. Tetrahedron Lett. 2018, 59, 1159.

[9] Zaki, Y. H.; Sayed, A. R.; Elroby, S. A. Chem. Cent. J. 2016, 10, 17.

[10] Suresh, G.; Nadh, R. V.; Srinivasu, N.; Kaushal, K. Synth. Commun. 2016, 46, 1972.

[11] Ning, G. H.; Zhao, W. T.; Bian, Q.; Tang, X. Y. Chin. J. Org. Chem. 2014, 34, 1800 (in Chinese). (宁国慧, 赵温涛, 边强, 唐向阳, 有机化学, 2014, 34, 1800.)

[12] Jiang, S.; Tsikolia, M.; Bernier, U. R.; Bloomquist, J. R. Int. J. Environ. Res. Public Health 2017, 14, 154.

[13] Filali, I.; Bouajila, J.; Znati, M.; Garah, B.-E.; Jannet, H. B. J. Med. Chem. 2015, 30, 371.

[14] Khazir, J.; Singh, P. P.; Reddy, D. M.; Hyder, I.; Shafi, S.; Sawant, S. D.; Chashoo, G.; Mahajan, A.; Alamc, M. S.; Saxena, A. K.; Arvinda, S.; Gupta, B. D.; Kumara, H. M. S. Eur. J. Med. Chem. 2013, 63, 279.

[15] Xue, C.; Wityak, J.; Sielecki, T. M.; Pinto, D. J.; Batt, D. G.; Cain, G. A.; Sworin, M.; Rockwell, A. L.; Roderick, J. J.; Wang, S.; Orwat, M. J.; Frietze, W. E.; Bostrom, L. L.; Liu, J.; Higley, C. A.; Rankin, F. W.; Tobin, A. E.; Emmett, G.; Lalka, G. K.; Sze, J. Y.; DiMeo, S. V.; Mousa, S. A.; Thoolen, M. J.; Racanelli, A. L.; Hausner, E. A.; Reilly, T. M.; DeGrado, W. F.; Wexler, R. R.; Olson, R. E. J. Med. Chem. 1997, 40, 2064.

[16] Cheng, K. F.; Al-Abed, Y. Bioorg. Med. Chem. Lett. 2006, 16, 3376.

[17] Goyard, D.; Kónya, B.; Chajistamatiou, A. S.; Chrysina, E. D.; Leroy, J.; Balzarin, S.; Tournier, M.; Tousch, D.; Petit, P.; Duret, C.; Maurel, P.; Somsák, L.; Docsa, T.; Gergely, P.; Praly, J.; Azay-Milhau, J.; Vidal, S. Eur. J. Med. Chem. 2016, 108, 444.

[18] (a) Talley, J. J.; Brown, D. L.; Carter, J. S.; Graneto, M. J.; Koboldt, C. M.; Masferrer, J. L.; Perkins, W. E.; Rogers, R. S.; Shaffer, A. F.; Zhang, Y. Y.; Zweifel, B. S.; Seibert, K. J. Med. Chem. 2000, 43, 775 .

(b) Talley, J. J.; Bertenshaw, S. R.; Brown, D. L.; Carter, J. S.; Graneto, M. J.; Kellogg, M. S.; Koboldt, C. M.; Yuan, J.; Zhang, Y. Y.; Seibert, K. J. Med. Chem. 2000, 43, 1661.

[19] Taylor, R. D.; MacCoss, M.; Lawson, A. D. G. J. Med. Chem. 2014, 57,5845 .

[20] (a) Minter, A. R.; Fuller, A. A.; Mapp, A. K. J. Am. Chem. Soc. 2003, 125,6846

(b) Fuller, A. A.; Chen, B.; Minter, A. R.; Mapp, A. K. J. Am. Chem. Soc. 2005, 127, 5376.

[21] Jiang, D.; Peng, J.; Chen, Y. Org. Lett. 2008, 10, 1695.

[22] Marotta, E.; Micheloni, E.; Scardovi, N.; Righi, P. Org. Lett. 2001, 3,727 .

[23] For selected reviews, see: (a) Kiss, L.; Nonn, M.; Fülöp, F. Synthesis 2012, 44, 1951.

(b) Vitale, P.; Scilimati, A. Synthesis 2013, 45, 2940.

[24] Yoshimura, A.; Middleton, K. R.; Todora, A. D.; Kastern, B. J.; Koski, S. R.; Maskaev, A. V.; Zhdankin, V. V. Org. Lett. 2013, 15, 4010.

[25] For selected examples on the cycloaddition of oximes withalkenes, see: (a) Zhu, M.-K.; Zhao, J.-F.; Loh, T.-P. J. Am. Chem. Soc. 2010, 132,6284 .

(b) Han, B.; Yang, X.-L.; Fang, R.; Yu, W.; Wang, C.; Duan, X.-Y.; Liu, S. Angew. Chem., Int. Ed. 2012, 51, 8816.

(c) Schmidt, E. Y.; Tatarinova, I. V.; Ivanova, E. V.; Zorina, N. V.; Ushakov, I. A.; Trofimov, B. A. Org. Lett. 2013, 15, 104.

(d) Dong, J.; Ding, T.; Zhang, S.; Chen, Z.; Tu, Y. Angew. Chem., 
Int. Ed. 2018, 57, 13192.

[26] For selected examples on the cycloaddition of nitro com-pounds with alkenes, see: (a) Cecchi, L.; DeSarlo, F.; Machetti, F. Chem.-Eur. J. 2008, 14, 7903.

(b) Trogu, E.; DeSarlo, F.; Machetti, F. Chem.-Eur. J. 2009, 15, 7940

(c) Chary, R. G.; Reddy, G. R.; Ganesh, Y. S. S.; Prasad, K. V.; Raghunadh, A.; Krishna, T.; Mukherjee, S.; Pal, M. Adv. Synth. Catal. 2014, 356, 160.

[27] Han, B.; Yang, X.-L.; Fang, R.; Yu, W.; Wang, C.; Duan, X.-Y.; Liu, S. Angew. Chem., Int. Ed. 2012, 51, 8816.

[28] For reviews, see: (a) Zard, S. Z. Chem. Soc. Rev. 2008, 37, 1603.

(b) Hartung, J. Eur. J. Org. Chem. 2001, 4, 619.

(c) Jasperse, C. P.; Curran, D. P.; Fevig, T. L. Chem. Rev. 1991, 91, 1237.

(d) Song, L.; Liu, K.; Li, C. Org. Lett. 2011, 13, 3434.

(e) Liu, F.; Liu, K.; Yuan, X.; Li, C. J. Org. Chem. 2007, 72, 10231.

[29] Jiang, D. H.; Peng, J. S.; Chen, Y. W. Org. Lett. 2008, 10, 1695.

[30] Zhu, M.-K.; Zhao, J.-F.; Loh, T.-P. J. Am. Chem. Soc. 2010, 132, 6284.

[31] Dong, K.-Y.; Qin, H.-T.; Bao, X.-X.; Liu, F.; Zhu, C. Org. Lett. 2014, 16, 5266

[32] For selected reviews on oxidation reactions with molecular oxygen, see: (a) Mukaiyama, T.; Yamada, T. Bull. Chem. Soc. Jpn. 1995, 68, 17.

(b) Punniyamurthy, T.; Velusamy, S.; Iqbal, J. Chem. Rev. 2005, 105, 2329.

(c) Piera, J.; Bäckvall, J.-E. Angew. Chem., Int. Ed. 2008, 47, 3506. (d) Wendlandt, A. E.; Suess, A. M.; Stahl, S. S. Angew. Chem., Int. Ed. 2011, 50, 11062 .

(e) Shi, Z.; Zhang, C.; Tang, C.; Jiao, N. Chem. Soc. Rev. 2012, 41, 3381

[33] (a) Bag, R.; Sar, D.; Punniyamurthy, T. Org. Lett. 2015, 17, 2010.

(b) Andia, A. A.; Miner, M. R.; Woerpel, K. A. Org. Lett. 2015, 17, 2704.

(c) Lu, Q.; Liu, Z.; Luo, Y.; Zhang, G.; Huang, Z.; Wang, H.; Liu, C.; Miller, J. T.; Lei, A. Org. Lett. 2015, 17, 3402.

(d) Lu, Q.; Peng, P.; Luo, Y.; Zhao, Y.; Zhou, M.; Lei, A. Chem.Eur. J. 2015, 21, 18580 .

(e) Xia, X.-F.; Zhu, S.-L.; Gu, Z.; Wang, H.; Li, W.; Liu, X.; Liang, Y.-M. J. Org. Chem. 2015, 80, 5572.

(f) Zhang, J.-Z.; Tang, Y. Adv. Synth. Catal. 2016, 358, 752.

(g) Miner, M. R.; Woerpel, K. A. Eur. J. Org. Chem. 2016, 34, 1860

(h) Bag, R.; Sar, D.; Punniyamurthy, T. Org. Biomol. Chem. 2016, 14,3246 .

[34] For the metal-free aerobic dioxygenation of $\mathrm{C}=\mathrm{C}$ bonds using hydrox-amic acids, see: (a) Schmidt, V. A.; Alexanian, E. J. Angew. Chem., Int. Ed. 2010, 49, 4491.

(b) Giglio, B. C.; Schmidt, V. A.; Alexanian, E. J. J. Am. Chem. Soc. 2011, 133, 13320

(c) Schmidt, V. A.; Alexanian, E. J. Chem. Sci. 2012, 3, 1672.

[35] For the aerobic oxytrifluoromethylation of $\mathrm{C}=\mathrm{C}$ bonds, see: (a) Zhang, C.-P.; Wang, Z.-L.; Chen, Q.-Y.; Zhang, C.-T.; Gu, Y.-C.; Xiao, J.-C. Chem. Commun. 2011, 47, 6632.

(b) Deb, A.; Manna, S.; Modak, A.; Patra, T.; Maity, S.; Maiti, D. Angew. Chem., Int. Ed. 2013, 52, 9747.

(c) Yang, Y.; Liu, Y.; Jiang, Y.; Zhang, Y.; Vicic, D. J. Org. Chem. 2015, 80, 6639 .

(d) Liu, C.; Lu, Q.; Huang, Z.; Zhang, J.; Liao, F.; Peng, P.; Lei, A. Org. Lett. 2015, 17, 6034 .

[36] For the aerobic oxy-2,2,2-trifluoroethylation of $\mathrm{C}=\mathrm{C}$ bonds, see: Li, L.; Huang, M.; Liu, C.; Xiao, J.-C.; Chen, Q.-Y.; Guo, Y.; Zhao, Z.-G. Org. Lett. 2015, 17, 4714.

[37] For the aerobic oxysulfonylation of $\mathrm{C}=\mathrm{C}$ bonds, see: (a) $\mathrm{Lu}, \mathrm{Q}$;; Zhang, J.; Wei, F.; Qi, Y.; Wang, H.; Liu, Z.; Lei, A. Angew. Chem., Int. Ed. 2013, 52, 7156.

(b) Wei, W.; Liu, C.; Yang, D.; Wen, J.; You, J.; Suo, Y.; Wang, H.
Chem. Commun. 2013, 49, 10239.

[38] Keshari, T.; Ya-dav, V. K.; Srivastava, V. P.; Yadav, L. D. S. Green Chem. 2014, 16, 3986.

[39] Zhou, S.-F.; Pan, X.; Zhou, Z.-H.; Shoberu, A.; Zou, J.-P. J. Org. Chem. 2015, 80, 3682.

[40] Wei, W.; Ji, J.-X. Angew. Chem., Int. Ed. 2011, 50, 9097.

[41] Sun, X.; Li, X.; Song, S.; Zhu, Y.; Liang, Y.-F.; Jiao, N. J. Am. Chem. Soc. 2015, 137, 6059.

[42] For autoxidative 4,5-dihydroisoxazoline ring formation through thenitrooxylation of $\mathrm{C}=\mathrm{C}$ bonds using stoichiometric amounts of tert-butylnitrite, see: Zhang, X.-W.; Xiao, Z.-F.; Zhuang, Y.-J.; Wang, M.-M.; Kang, Y.-B. Adv. Synth. Catal. 2016, 358, 1942.

[43] (a) Castellano, S.; Kuck, D.; Vivi-ano, M.; Yoo, J.; López-Vallejo, F.; Conti, P.; Tamborini, L.; Pinto, A.; Medina-Franco, J. L.; Sbardella, G. J. Med. Chem. 2011, 54, 7663.

(b) Patel, N. C.; Schwarz, J.; Hou, X. J.; Hoover, D. J.; Xie, L.; Fliri, A. J.; Gallaschun, R. J.; Lazzaro, J. T.; Bryce, D. K.; Hoffmann, W. E.; Hanks, A. N.; McGinnis, D.; Marr, E. S.; Gazard, J. L.; Hajós, M.; Scialis, R. J.; Hurst, R. S.; Shaffer, C. L.; Pandit, J.; O'Donnell, C. J. J. Med. Chem. 2013, 56, 9180.

(c) Rodrigues, G. C.; Feijó, D. F.; Bozza, M. T.; Pan, P.; Vullo, D.; Parkkila, S.; Supuran, C. T.; Capasso, C.; Aguiar, A. P.; Vermelho, A. B. J. Med. Chem. 2014, 57, 298.

[44] (a) Nishikawa, N. Method and Applications of Cycloaddition Reactions in Organic Syntheses, Wiley, Hoboken, NJ, 2014, pp. 565 598.

(b) Feuer, H. Nitrile Oxides, Nitrones, and Nitronates in Organic Synthesis: Novel Strategies in Synthesis, Wiley, Hoboken, NJ, 2008, p. 760 .

(c) Kotyatkina, A. I.; Zhabinsky, V. N.; Khripach, V. A. Russ. Chem. Rev. 2001, 70, 641

(d) Kozikowski, A. P. Acc. Chem. Res. 1984, 17, 410.

[45] Yamamoto, D.; Oguro, T.; Tashiro, Y.; Soga, M.; Miyashita, K.; Aso, Y.; Makino, K. Eur. J. Org. Chem. 2016, 31, 5216.

[46] Yamamoto, D.; Soga, M.; Ansai, H.; Makino, K. Org. Chem. Front. 2016, 3, 1420 .

[47] (a) Shcroft, C. P.; Hellier, P.; Pettman, A.; Wakinson, S. Org. Process Res. Dev. 2011, 15, 98.

(b) Ettari, R.; Nizi, E.; Franceco, M. E. D.; Dude, M.-A.; Pradel, G.; Vičík, R.; Schirmeister, T.; Micale, N.; Grasso, S.; Zappalà, M. $J$. Med. Chem. 2008, 51, 988.

(c) Reck, F.; Zhou, F.; Girardot, M.; Kern, G.; Eyermann, C. J.; Hales, N. J.; Ramsay, R. R.; Gravestock, M. B. J. Med. Chem. 2005, 48, 499.

[48] (a) Sun, X.; Yu, F.; Ye, T.; Liang, X.; Ye, J. Chem.-Eur. J. 2011, 17, 430.

(b) El-Awa, A.; Noshi, M. N.; du Jourdin, X. M.; Fuchs, P. L. Chem. Rev. 2009, 109, 2315.

(c) Plesniak, K.; Zarecki, A.; Wicha, J. Top. Curr. Chem. 2007, 275, 163.

[49] Gao, Y.; Tang, X.; Peng, J.; Hu, M.; Wu, W.; Jiang, H. Org. Lett. 2016, 18, 1158.

[50] Wang, L.-J.; Chen, M.-M.; Qi, L.; Xu, Z.-D.; Li, W. Chem. Commun. 2017, 52, 2056.

[51] (a) Li, Y.; Studer, A. Angew. Chem., Int. Ed. 2012, 51, 8221. (b) Zhang, B.; Studer, A. Org. Lett. 2013, 15, 4548.

[52] Lv, Y. H.; Pu, W. Y.; Wang, Q. Q.; Chen, Q.; Niu, J. J.; Zhang, Q. Adv. Synth. Catal. 2017, 359, 3114.

[53] Sun, K.; Li, G. F.; Li, Y. Y.; Yu, J.; Zhao, Q.; Zhang, Z. G.; Zhang, G. S. Adv. Synth. Catal. 2020, 362, 10.

[54] Wang, X.; Li, G. F.; Sun, K.; Zhang, B. Chin. J. Org. Chem. 2020 40,913 (in Chinese).

(王薪, 李国锋, 孙凯, 张冰, 有机化学, 2020, 40,913.)

[55] Sun, K.; Wang, S. N.; Feng, R. R.; Zhang, Y. X.; Wang, X.; Zhang, Z. G.; Zhang, B. Org. Lett. 2019, 21, 2052.

[56] Liu, X.-Y.; Li, X.-T.; Gu, Q.-S.; Dong, X.-Y.; Meng, X. Angew. Chem., Int. Ed. 2018, 57, 7668 .

[57] (a) Zhu, R.; Buchwald, S. L. Angew. Chem., Int. Ed. 2013, 52, 
12655.

(b) Zhu, R.; Buchwald, S. L. J. Am. Chem. Soc. 2015, 137, 8069

(c) Zhang, W.; Wang, F.; McCann, S. D.; Wang, D.; Chen, P.; Stahl, S. S.; Liu, G. Science 2016, 353, 1014.

(d) Wang, F.; Wang, D.; Wan, X.; Wu, L.; Chen, P.; Liu, G. J. Am. Chem. Soc. 2016, 138, 15547.

(e) Wang, D.; Wang, F.; Chen, P.; Lin, Z.; Liu, G. Angew. Chem., Int. Ed. 2017, 56, 2054.

(f) Wu, L.; Wang, F.; Wan, X.; Wang, D.; Chen, P.; Liu, G. J. Am. Chem. Soc. 2017, 139, 2904.

[58] (a) Lin, J.-S.; Dong, X.-Y.; Li, T.-T.; Jiang, N.-C.; Tan, B.; Liu, X.-Y. J. Am. Chem. Soc. 2016, 138, 9357.

(b) Lin, J.-S.; Wang, F.-L.; Dong, X.-Y.; He, W.-W.; Yuan, Y.; Chen, S.; Liu, X.-Y. Nat. Commun. 2017, 8, 14841 .

(c) Cheng, Y.-F.; Dong, X.-Y.; Gu, Q.-S.; Yu, Z.-L.; Liu, X.-Y. Angew. Chem., Int. Ed. 2017, 56, 8883.

(d) Wang, F.-L.; Dong, X.-Y.; Lin, J.-S.; Zeng, Y.; Jiao, G.-Y.; Gu, Q.-S.; Guo, X.-Q.; Ma, C.-L.; Liu, X.-Y. Chem. Commun. 2017, 3, 979.

[59] For selected references on $\mathrm{Cu}(\mathrm{II})-\mathrm{O}(\mathrm{N})$ species and reactions involvingalkyl radical intermediates, see: (a) Huffman, L. M.; Stahl, S. S. J. Am. Chem. Soc. 2008, 130, 9196.

(b) Creutz, S. E.; Lotito, K. J.; Fu, G. C.; Peters, J. C. Science 2012, $338,647$.

(c) Tran, B. L.; Li, B.; Driess, M.; Hartwig, J. F. J. Am. Chem. Soc. 2014, 136, 2555.

[60] For selected examples, see: (a) He, Y.-T.; Li, L.-H.; Yang, Y.-F.; Wang, Y.-Q.; Luo, J.-Y.; Liu, X.-Y.; Liang, Y.-M. Chem. Commun. 2013, 49, 5687.

(b) Wei, Q.; Chen, J.-R.; Hu, X.-Q.; Yang, X.-C.; Lu, B.; Xiao, W.-J. Org. Lett. 2015, 17, 4464.

(c) Zhang, W.; Su, Y.; Wang, K.-H.; Wu, L.; Chang, B.; Shi, Y.; Huang, D.; Hu, Y. Org. Lett. 2017, 19, 376

[61] Mukaiyama, T.; Yamada, T. Bull. Chem. Soc. Jpn. 1995, 68, 17.

[62] Li, W.; Jia, P.; Han, B.; Li, D.; Yu, W. Tetrahedron 2013, 69, 3274.

[63] Peng, X.-X.; Deng, Y.-J.; Yang, X.-L.; Zhang, L.; Yu, W.; Han, B. Org. Lett. 2014, 16, 4650.

[64] (a) Chen, Y.-X.; Qian, L.-F.; Zhang, W.; Han, B. Angew. Chem. 2008, $120,9470$.

(b) Han, B.; Wang, C.; Han, R.-F.; Yu, W.; Duan, X.-Y.; Fang, R.; Yang, X.-L. Chem. Commun. 2011, 47, 7818.

(c) Han, B.; Yang, X.-L.; Wang, C.; Bai, Y.-W.; Pan, T.-C.; Chen, X.; Yu, W. J. Org. Chem. 2012, 77, 1136.

(d) Han, B.; Han, R.-F.; Ren, Y.-W.; Duan, X.-Y.; Xu, Y.-C.; Zhang, W. Tetrahedron 2011, 67, 5615.

[65] (a) Pratt, D. A.; Blake, J. A.; Mulder, P.; Walton, J. C.; Korth, H.-G.; Ingold, K. U. J. Am. Chem. Soc. 2004, 126, 10667.

(b) Chong, S.-S.; Fu, Y.; Liu, L.; Guo, Q.-X. J. Phys. Chem. A 2007, 111, 13112.

[66] For transition metal catalyzed vicinal dioxygenation, oxyamination, and diamination of olefins, see: (a) Kolb, H. C.; VanNieuwenhze, M. S.; Sharpless, K. B. Chem. Rev. 1994, 94, 2483.

(b) Wang, A.; Jiang, H.; Chen, H. J. Am. Chem. Soc. 2009, 131, 3846 .

(c) Zhang, Y.; Sigman, M. S. J. Am. Chem. Soc. 2007, 129, 3076.

(d) Fuller, P. H.; Kim, J.-W.; Chemler, S. R. J. Am. Chem. Soc. 2008, 130,17638

(e) Williamson, K. S.; Yoon, T. P. J. Am. Chem. Soc. 2010, 132, 4570 .

(f) Du, H.; Zhao, B.; Shi, Y. J. Am. Chem. Soc. 2008, 130, 8590.

[67] Selected examples of biological evaluation of isoxazolines: (a) Castellano, S.; Kuck, D.; Viviano, M.; Yoo, J.; López-Vallejo, F.; Conti, P.; Tamborini, L.; Pinto, A.; Medina-Franco, J. L.; Sbardella, G. J. J. Med. Chem. 2011, 54, 7663.

(b) Poutiainen, P. K.; Venäläinen, T. A.; Peräkylä, M.; Matilainen, J. M.; Väisänen, S.; Honkakoski, P.; Laatikainen, R.; Pulkkinen, J. T. Bioorg. Med. Chem. 2010, 18, 3437.

(c) Bode, J. W.; Carreira, E. M. Org. Lett. 2001, 3, 1587 . (d) Curran, D. P.; Heffner, T. A. J. Org. Chem. 1990, 55, 4585

(e) Kozikowski, A. P. Acc. Chem. Res. 1984, 17, 410.

(f) Jiang, D.; Peng, J.; Chen, Y. Org. Lett. 2008, 10, 1695.

(g) Zhu, M.-K.; Zhao, J.-F.; Loh, T.-P. J. Am. Chem. Soc. 2010, 132, 6284.

(h) He, Y.-H.; Li, L.-H.; Yang, Y.-F.; Wang, Y.-Q.; Luo, J.-Y.; Liu, X.-Y.; Liang, Y.-M. Chem. Commun. 2013, 49, 5687.

(i) Tripathi, C. B.; Mukherjee, S. Angew. Chem., Int. Ed. 2013, 52, 8450 .

[68] Some selected reviews: (a) Zhdankin, V. V.; Stang, P. J. Chem. Rev. 2002, 102, 2523

(b) Zhdankin, V. V.; Stang, P. J. Chem. Rev. 2008, 108, 5299.

(c) Schafer, S.; Wirth, T. Angew. Chem., Int. Ed. 2010, 49, 2786.

(d) Dohi, T.; Kita, Y. Chem. Commun. 2009, 16, 2073.

(e) Ngatimin, M.; Lupton, D. W. Aust. J. Chem. 2010, 63, 653.

(f) Uyanik, M.; Ishihara, K. Chem. Commun. 2009, 16, 2086.

(g) Ciufolini, M. A.; Braun, N. A.; Canesi, S.; Ousmer, M.; Chang, J.; Chai, D. Synthesis 2007, 3759.

[69] Some selected examples where polyvalent iodine reagents mediate alkene difunctionalization:

(a) Lovick, H. M.; Michael, F. E. J. Am. Chem. Soc. 2010, 132, 1249 .

(b) Wardrop, D. J.; Bowen, E. G.; Forslund, R. E.; Sussman, A. D.; Weerasekera, S. L. J. Am. Chem. Soc. 2010, 132, 1188.

(c) Kang, Y.-B.; Gade, L. H. J. Am. Chem. Soc. 2011, 133, 3658

(d) Röben, C.; Souto, J. A.; Gonzálz, Y.; Lishchynskyi, A.; Muńiz, K. Angew. Chem., Int. Ed. 2011, 50, 9478.

(e) Cochran, B. M.; Michael, F. E. Org. Lett. 2008, 10, 5039.

(f) Correa, A.; Tellitu, I.; Domínguez, E.; Sanmartin, R. J. Org Chem. 2006, 71, 8316 .

(g) Muńiz, K.; Hövelmann, C. H.; Campos-Gómez, E.; Barluenga, J.; González, J. M.; Streuff, J.; Nieger, M. Chem.-Asian J. 2008, 3, 776. (h) Li, H.; Widenhoefer. Tetrahedron 2010, 66, 4827.

(i) Fujita, M.; Wakita, W.; Sugimura, T. Chem. Commun. 2011, 47, 3983.

[70] (a) Müller, K.; Faeh, C.; Diederich, F. Science 2007, 317, 1881.

(b) Purser, S.; Moore, P. R.; Swallow, S.; Gouverneur, V. Chem. Soc. Rev. 2008, 37, 320.

[71] Kong, W.; Guo, Q.; Xu, Z.; Wang, G.; Jiang, X.; Wang, R. Org. Lett. 2015, 17, 3686

[72] Yu, J.-M.; Cai, C. Org. Biomol. Chem. 2018, 16, 490.

[73] (a) Chen, F.; Yang, X. L.; Wu, Z. W.; Han, B. J. Org. Chem. 2016, 81,3042 .

(b) Duan, X. Y.; Yang, X. L.; Fang, R.; Peng, X. X.; Yu, W.; Han, B. J. Org. Chem. 2013, 78, 10692.

[74] Peng, X. X.; Deng, Y. J.; Yang, X. L.; Zhang, L.; Yu, W.; Han, B. Org. Lett. 2014, 16, 4650.

[75] Yang, X. L.; Chen, F.; Zhou, N. N.; Yu, W.; Han, B. Org. Lett. 2014, $16,6476$.

[76] (a) Kong, W.; Guo, Q.; Xu, Z.; Wang, G.; Jiang, X.; Wang, R. Org. Lett. 2015, 17, 3686.

(b) Hu, X. Q.; Feng, G.; Chen, J. R.; Yan, D. M.; Zhao, Q. Q.; Wei, Q.; Xiao, W. J. Org. Biomol. Chem. 2015, 13, 3457.

[77] Zhang, X.-W.; Xiao, Z.-F.; Zhuang, Y.-J.; Wang, M.-M.; Kang, Y.-B. Adv. Synth. Catal. 2016, 358, 1942.

[78] For iminoxyl radicals proved by EPR spectroscopy orother methods, see: (a) Zhu, X.; Wang, Y.-F.; Ren, W.; Zhang, F.-L.; Chiba, S. Org. Lett. 2013, 15, 3214.

(b) Liu, Y.-Y.; Yang, X.-H.; Yang, J.; Song, R.-J.; Li, J.-H. Chem. Commun. 2014, 50, 6906.

(c) Krylov, I. B.; Terentev, A. O.; Timofeev, V. P.; Shelimov, B. N.; Novikov, R. A.; Merkulova, V. M.; Nikishin, G. I. Adv. Synth. Catal. 2014, 356, 2266

(d) Yang, X.-L.; Chen, F.; Zhou, N.-N.; Yu, W.; Han, B. Org. Lett. 2014, 16, 6476.

(e) Lemercier, B. C.; Pierce, J. G. Org. Lett. 2015, 17, 4542.

[79] Peng, X.-X.; Deng, Y.-J.; Yang, X.-L.; Zhang, L.; Yu, W.; Han, B. Org. Lett. 2014, 16, 4650. 
[80] (a) Galliker, B.; Kissner, R.; Nauser, T.; Koppenol, W. H. Chem.-Eur. J. 2009, 15, 6161.

(b) Taniguchi, T.; Yajima, A.; Ishibashi, H. Adv. Synth. Catal. 2011, 353, 2643.

[81] Lopes, E. F.; Penteado, F.; Thurow, S.; Pinz, M.; Reis, A.; Wilhelm, E.; Luchese, C.; Barcellos, T.; Dalberto, B.; Alves, D.; Silva, M. S. D.; Lenardão, E. J. Org. Chem. 2019, 84, 12452.

[82] Triandafillidi, I.; Kokotos, C. G. Org. Lett. 2017, 19, 4254.

[83] (a) Limnios, D.; Kokotos, C. G. ACS Catal. 2013, 3, 2239.

(b) Limnios, D.; Kokotos, C. G. Chem.-Eur. J. 2014, 20, 559.

(c) Limnios, D.; Kokotos, C. G. J. Org. Chem. 2014, 79, 4270.

(d) Theodorou, A.; Limnios, D.; Kokotos, C. G. Chem.-Eur. J. 2015, 21,5238 .

[84] Zhang, W. G.; Su, Y. P.; Wang, K.-H.; Wu, L. L.; Chang, B. B.; Shi, Y.; Huang, D. F.; Hu, Y. L. Org. Lett. 2017, 19, 376.

[85] (a) Tilstam, U.; Weinmann, H. Org. Process Res. Dev. 2002, 6, 384. (b) Mendonça, G. F.; Sindra, H. C.; de Almeida, L. S.; Esteves, P. M.; de Mattos, M. C. S. Tetrahedron Lett. 2009, 50, 473.

(c) Mishra, A. K.; Nagarajaiah, H.; Moorthy, J. N. Eur. J. Org. Chem. 2015, 46, 2733.

(d) Gaspa, S.; Porcheddu, A.; Luca, L. D. Adv. Synth. Catal. 2016, $358,154$.

[86] (a) Luca, L. D.; Giacomelli, G.; Porcheddu, A. Org. Lett. 2001, 3, 3041.

(b) Ye, J.; Wang, Y.; Chen, J.; Liang, X. Adv. Synth. Catal. 2004, 346, 691.

(c) Sniady, A.; Morreale, M. S.; Wheeler, K. A.; Dembinski, R. Eur. J. Org. Chem. 2008, 39, 3449.

(d) Jing, Y.; Daniliuc, C. G.; Studer, A. Org. Lett. 2014, 16, 4932.

(e) Raihan, M. J.; Rajawinslin, R. R.; Kavala, V.; Kuo, C.-W.; Kuo, T.-S.; He, C.-H.; Huang, H.-N.; Yao, C.-F. J. Org. Chem. 2013, 78, 8872.

[87] (a) Morimoto, H.; Tsubogo, T.; Litvinas, N. D.; Hartwig, J. F. Angew. Chem., Int. Ed. 2011, 50, 3793.

(b) Litvinas, N. D.; Fier, P. S.; Hartwig, J. F. Angew. Chem., Int. Ed. 2012, 51, 536 .

(c) Oishi, M.; Kondo, H.; Amii, H. Chem. Commun. 2009, 14, 1909.

(d) Weng, Z.; Lee, R.; Jia, W.; Yuan, Y.; Wang, W.; Feng, X.;
Huang, K.-W. Organometallics 2011, 30, 3229.

(e) Shimizu, N.; Kondo, H.; Oishi, M.; Fujikawa, K.; Komoda, K.; Amii, H. Org. Synth. 2016, 93, 147.

[88] Wei, Q.; Chen, J.-R.; Hu, X.-Q.; Yang, X.-C.; Lu, B.; Xiao, W.-J. Org. Lett. 2015, 17, 4464.

[89] For reviews of difluoromethylation: (a) Rong, J.; Ni, C.; Hu, J. Asian J. Org. Chem. 2017, 6, 139.

(b) Belhomme, M.-C.; Besset, T.; Poisson, T.; Pannecoucke, X. Chem.-Eur. J. 2015, 21, 12836.

(c) Yerien, D. E.; Barata-Vallejo, S.; Postigo, A. Chem.-Eur. J. 2017, 23, 14676

[90] (a) Fujiwara, Y.; Dixon, J. A.; O'Hara, F.; Funder, E. D.; Dixon, D. D.; Rodri-guez, R. A.; Baxter, R. D.; Herle, B.; Sach, N.; Collins, M. R.; Ishihara, Y.; Baran, P. S. Nature 2012, 492, 95.

(b) Fujiwara, Y.; Dixon, J. A.; Rodriguez, R. A.; Baxter, R. D.; Dixon, D. D.; Collins, M. R.; Blackmond, D. G.; Baran, P. S. J. Am. Chem. Soc. 2012, 134, 1494.

[91] For selected examples, see: (a) Lin, Q.-Y.; Xu, X.-H.; Zhang, K.; Qing, F.-L. Angew. Chem., Int. Ed. 2016, 55, 1479.

(b) He, Z.; Tan, P.; Ni, C.; Hu, J. Org. Lett. 2015, 17, 1838.

(c) Arai, Y.; Tomita, R.; Ando, G.; Koike, T.; Akita, M. Chem.-Eur. J. 2016, 22, 1262.

(d) Zou, G.; Wang, X. Org. Biomol. Chem. 2017, 15, 8748.

(e) Ran, Y.; Lin, Q.-Y.; Xu, X.-H.; Qing, F.-L. J. Org. Chem. 2016, 81,7001 .

(f) Tang, X.-J.; Dolbier, W. R. Angew. Chem., Int. Ed. 2015, 54 , 4246.

(g) Noto, N.; Koike, T.; Akita, M. J. Org. Chem. 2016, 81, 7064

(h) Tang, X.; Zhang, Z.; Dolbier, W. R. Chem.-Eur. J. 2015, 21, 18961.

(i) Lin, Q.-Y.; Ran, Y.; Xu, X.-H.; Qing, F.-L. Org. Lett. 2016, 18 , 2419.

[92] Noto, N.; Koike, T.; Akita, M. Chem. Sci. 2017, 8, 6375.

[93] Xiong, P.; Xu, H.-H.; Song, J. S.; Xu, H.-C. J. Am. Chem. Soc. 2018, 140, 2460.

[94] Zhu, M.; Fun, W. J.; Guo, W. B.; Tian, Y. F.; Wang, Z. Q.; Xu, C.; Ji, B. M. Eur. J. Org. Chem. 2019, 14, 1614.

[95] Xiao, W.-J.; Hu, X.-Q.; Chen, J.; Chen, J.-R.; Yan, D.-M. Chem.-Eur. J. 2016, 22, 14141. 